\title{
Either fadD1 or fadD2, Which Encode acyl-CoA Synthetase, Is Essential for the Survival of Haemophilus parasuis SC096
}

\author{
Saixiang Feng ${ }^{1}$, Chenggang $X u^{1}$, Kaijie Yang ${ }^{1}$, Haihong Wang ${ }^{2}$, Huiying Fan ${ }^{1 *}$ and \\ Ming Liao ${ }^{1 *}$ \\ ${ }^{1}$ Key Laboratory of Veterinary Vaccine Innovation of the Ministry of Agriculture, College of Veterinary Medicine, South China \\ Agricultural University, Guangzhou, China, ${ }^{2}$ Key Laboratory of Protein Function and Regulation in Agricultural Organisms of \\ Guangdong province, College of Life Science, South China Agricultural University, Guangzhou, China
}

In Haemophilus parasuis, the genes HAPS_0217 and HAPS_1695 are predicted to encode long-chain fatty acid-CoA ligases (FACSs). These proteins contain ATP/AMP signature motifs and FACS conserved motifs that are homologous to those in Escherichia coli FadD (EcFadD). In this study, we demonstrate that HAPS_0217 and HAPS_1695 can functionally replace EcFadD in the E. coli fadD mutant JW1794, and were thus designated fadD1 and fadD2, respectively. An evaluation of kinetic parameters indicated

OPEN ACCESS

Edited by:

Margaret E. Bauer,

Indiana University School of Medicine,

USA

Reviewed by:

Jianjun Sun,

University of Texas at EI Paso, USA

Huafeng Wang,

University of Wisconsin-Madison, USA

Francis Alonzo,

Loyola University Chicago, USA

*Correspondence:

Huiying Fan

fanhy@scau.edu.cn

Ming Liao

mliao@scau.edu.cn

Received: 28 December 2016

Accepted: 27 February 2017

Published: 15 March 2017

Citation:

Feng S, Xu C, Yang K, Wang H, Fan H and Liao M (2017) Either fadD1 or fadD2, Which Encode acyl-CoA Synthetase, Is Essential for the Survival of Haemophilus parasuis

Front. Cell. Infect. Microbiol. 7:72. doi: 10.3389/fcimb.2017.00072 that FadD1 and FadD2 have a substrate preference for long-chain fatty acids. Moreover, FadD2 exhibited substrate inhibition in the presence of high concentrations of oleic acid. Single mutants of each of the fadD genes were easily constructed, whereas double mutants were not. These results were further confirmed using genomic site-directed mutagenesis, which supported the idea that $H$. parasuis requires either fadD1 or fadD2 for survival. The fadD1 mutant exhibited slower growth than the wild-type strain SC096, and its complementation resulted in a restored phenotype. The wild-type strain did not grow on chemically defined medium without the addition of oleic acid, indicating that lipids are a vital nutrient for this bacterium. Additionally, strains with a disrupted fadD1 gene also exhibited increased sensitivity to quinolone antibiotics, including levofloxacin, enrofloxacin, ciprofloxacin and nalidixic acid.

Keywords: Haemophilus parasuis, acyl-CoA synthetase, FadD, fatty acid, quinolones

\section{INTRODUCTION}

Haemophilus parasuis is a pathogenic bacterium of the upper respiratory tract in conventional pigs and the etiological agent of Glässer's disease, which is characterized by fibrinous polyserositis, arthritis, and meningitis. H. parasuis has emerged as a major cause of high mortality in swine that contributes to large economic losses in the pig industry worldwide (Oliveira and Pijoan, 2004). Intensive studies have attempted to identify the virulence-associated genes in $H$. parasuis (Costa-Hurtado and Aragon, 2013). However, several of the specific mechanisms underlying its pathogenicity remain to be investigated. Previous differential expression studies showed that exogenous fatty acid utilization enzyme FadD was a potential virulence factor in $H$. parasuis (Hill et al., 2003; Metcalf and MacInnes, 2007). Fatty acids are important metabolic intermediates as well as major components of phospholipids, which are essential for membrane formation 
in pathogenic bacteria (Zhang and Rock, 2008a). Because fatty acid biosynthesis is vital and energetically expensive, most pathogens use and incorporate extracellular fatty acids into their phospholipid membrane (Yao and Rock, 2015).

Over the past five decades, the fatty acid synthesis (FAS) and exogenous fatty acid incorporation pathways have been fully characterized in Escherichia coli (Rock and Jackowski, 2002). A highly conserved set of genes encode the enzymes that perform each of the individual steps in the FAS pathway (White et al., 2005). Alternatively, cells initiate the use of extracellular fatty acids by forming fatty acyl coenzyme A (acylCoA) using long-chain acyl-CoA ligase (FACS) (Black and DiRusso, 2003). Subsequently, phospholipids can be directly synthesized by acyltransferases using acyl-CoA (Zhang and Rock, 2008b). Another pathway uses acyl-CoA as a carbon source via $\beta$-oxidation (Weeks et al., 1969). Previous studies have shown that exogenous fatty acids are important participants in bacterial invasion and infection (DiRusso et al., 1999). For example, host cell phospholipase is required for Salmonella enterica serovar Typhimurium to invade epithelial cells (Falkow et al., 1992). Fatty acids are released by phospholipase and then imported and activated by the bacterial exogenous fatty acid transport system, resulting in an increase in the utilization of long-chain acyl-CoA (Pace et al., 1993).

The product of the $f a d D$ gene is a long-chain fatty acyl-CoA ligase that converts exogenous long-chain fatty acids (LCFAs) into acyl-CoA in bacteria (Black et al., 1992). In Vibrio cholerae, disrupting $f a d D$ results in attenuated virulence. In the $f a d D$ mutant, the expression of the major virulence genes in the Tox $\mathrm{R}$ regulon is repressed, and the membrane localization of the master regulator TcpP is impaired (Ray et al., 2011). The fadD mutant of $S$. enterica serovar Typhi has reduced hilA expression and invasiveness in HEp-2 cells, where hilA is a transcriptional regulator that is responsible for directly regulating the expression of genes, such as those involved in the type III secretory apparatus, that are required for invasiveness in $S$. enterica serovar Typhi (Lucas et al., 2000). Notably, fadD is not an essential gene for the survival of most bacteria, although it is important for pathogenic virulence activity in some bacteria. Pathogens can regulate FAS genes to supply endogenous fatty acids in an exogenous fatty acid-limited environment (DiRusso et al., 1999).

Here, we report the identification of two long-chain acylCoA ligases, FadD1 and FadD2, in H. parasuis SC096. These enzymes were confirmed to be responsible for the formation of long-chain fatty acyl-CoA. Either fadD1 or fadD2 is essential for the survival of $H$. parasuis SC096 grown in TSA medium containing bovine serum and NAD. The fadD1 mutant exhibited an impaired growth phenotype in a chemically defined medium and was more susceptible to quinolone antibiotics.

\section{MATERIALS AND METHODS}

\section{Bacterial Strains, Plasmids and Growth Conditions}

The bacterial strains and plasmids used in this study are listed in Table 1. E. coli was grown in LB medium (10 g/l tryptone, $5 \mathrm{~g} / \mathrm{l}$ yeast extract, and $10 \mathrm{~g} / \mathrm{l} \mathrm{NaCl} ; \mathrm{pH} 7.0)$ at $37^{\circ} \mathrm{C}$ following a routine protocol. $H$. parasuis was grown in Trypticase Soy Agar (TSA) (Oxoid, Hampshire, UK) supplemented with $0.002 \%$ nicotinamide adenine dinucleotide (NAD) (Sigma Aldrich, USA) and $5 \%$ inactivated bovine serum at $37^{\circ} \mathrm{C}$ in $5 \% \mathrm{CO}_{2}$. The chemically defined medium was prepared according to a previously described protocol (Murphy and Brauer, 2011) with some modifications and had the following

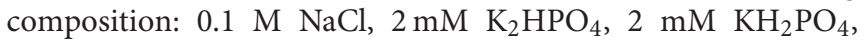
$4 \mathrm{mM} \mathrm{Na}_{2}$ EDTA, $4 \mathrm{mM} \mathrm{NH}_{4} \mathrm{Cl}, 0.125 \mathrm{mM} \mathrm{NaHCO}_{3}, 0.006 \mathrm{mM}$ thiamine hydrochloride, $0.001 \mathrm{mM}$ thiamine pyrophosphate, $0.008 \mathrm{mM}$ calcium pantothenate, $0.02 \%$ nicotinamide adenine dinucleotide, $0.375 \mathrm{mM}$ hypoxanthine, $0.45 \mathrm{mM}$ uracil, $0.15 \mathrm{mM}$ glutathione, $0.012 \mathrm{mM}$ biotin, $5 \%$ glucose, $0.1 \mathrm{mM} \mathrm{Fe}\left(\mathrm{NO}_{3}\right)$, $2.5 \mathrm{mM} \mathrm{MgCl}_{2}, 0.6 \mathrm{mM} \mathrm{CaCl}_{2}, 6.25 \mathrm{mM} \mathrm{Na}$ acetate trihydrate, $1.125 \mathrm{mM}$ L-alanine, $0.875 \mathrm{mM} \mathrm{L}$-arginine hydrochloride, 0.2 $\mathrm{mM}$ L-asparagine, $3.75 \mathrm{mM}$ L-aspartic acid, $0.35 \mathrm{mM} \mathrm{L}$ cysteine hydrochloride hydrate, $0.15 \mathrm{mM}$ L-cysteine, $7.5 \mathrm{mM}$ L-glutamic acid, $0.35 \mathrm{mM}$ L-glutamine, $0.225 \mathrm{mM}$ glycine, 0.10 $\mathrm{mM}$ L-histidine, $0.225 \mathrm{mM}$ L-isoleucine, $0.7 \mathrm{mM}$ L-leucine, $0.35 \mathrm{mM}$ L-lysine hydrochloride, $0.1 \mathrm{mM}$ L-methionine, 0.15 $\mathrm{mM}$ L-phenylalanine, $0.45 \mathrm{mM}$ L-proline, $0.475 \mathrm{mM}$ L-serine, $0.425 \mathrm{mM}$ L-threonine, $0.4 \mathrm{mM}$ L-tryptophan, $0.4 \mathrm{mM} \mathrm{L}$ tyrosine, and $0.525 \mathrm{mM} \mathrm{L}$-valine, $\mathrm{pH}$ 7.2. When required, the medium was supplemented with kanamycin $(30 \mu \mathrm{g} / \mathrm{ml})$ and gentamicin $(30 \mu \mathrm{g} / \mathrm{ml})$ to grow $E$. coli and $H$. parasuis or erythromycin $(10 \mu \mathrm{g} / \mathrm{ml})$ to grow $H$. parasuis alone. Bacterial growth was determined by measuring the optical density at $600 \mathrm{~nm}$.

\section{Complementation of an E. coli $\Delta$ fadD Strain}

A fadD mutant strain of E. coli (JW1794) was used for complementation studies (Baba et al., 2006). The $H$. parasuis $f a d D$ genes were amplified from the genomic DNA of the SC096 strain using the primers P1-P4, which contain NdeI and HindIII sites (as shown in Table 2). The PCR fragments were purified and cloned into the pMD19-T vector (Takara). The fadD sequences were confirmed by sequencing. The pSF226 (carrying fadD1) and pSF227 (carrying fadD2) plasmids were constructed by ligating NdeI-HindIII-digested fragments obtained from pMD19-T with the expression vector pBAD24m, which was digested with the same enzymes. The two recombinant vectors were introduced into the E. coli strain JW1794 for complementation. An empty vector was also transformed into JW1794 and used as a negative control in the complementation experiments. These strains were inoculated into M9 minimal medium supplemented with $0.1 \%$ fatty acid with Brij58 $(0.02 \%$ arabinose was added if required), and the complementation results were determined after the cells were incubated for $72 \mathrm{~h}$ at $37^{\circ} \mathrm{C}$.

\section{FadD Expression and Purification}

To produce the expression plasmids pSF228 (pET28b carrying fadD1) and pSF229 (pET28b carrying fadD2), cloning vectors (pMD19-T) carrying the above-constructed fadD genes were digested using NdeI and HindIII. The fragments were gel-purified and ligated into pET28b, which was digested using the same 
TABLE 1 | Bacterial strains and plasmids used in this study.

Strain or plasmid Relevant characteristic(s) Source

\section{E. coli strains}

\begin{tabular}{lll}
\hline DH5 $\alpha$ & $\mathrm{F}^{-}, \phi 80 \mathrm{~d} / \mathrm{lacZ} \Delta \mathrm{M} 15, \Delta($ lacZYA-argF)169, recA1, endA1, hsdR17, $\Delta$ phoA8, glnV44, deoR481, gyrA96 & Laboratory collection \\
BW25113 & $l a c / 9, r r n B_{\mathrm{T} 14}, \Delta / a c Z_{\mathrm{WJ16}}, h s d R 514, \Delta a r a B A D_{\mathrm{AH} 33}, \Delta r h a B A D_{\mathrm{LD} 78}$ & Datsenko and Wanner, 2000 \\
JW1794 & BW25113 $\Delta$ fadD::Kan & Baba et al., 2008 \\
BL21 (DE3) & $\mathrm{F}^{-}$, ompT, $h s d S_{\mathrm{B}}\left(\mathrm{r}_{\mathrm{B}}^{-} \mathrm{m}_{\mathrm{B}}^{-}\right)$, gal, dcm, (DE3) & Thermofisher \\
\hline
\end{tabular}

\section{H. parasuis strains}

\begin{tabular}{|c|c|}
\hline H. parasuis SC096 & Serovar 4 clinical isolate \\
\hline SF345 ( $\Delta$ fadD1) & SC096 $\Delta$ fadD1::Gm ${ }^{r}$ \\
\hline SF346 ( $\Delta$ fadD2) & SC096 $\Delta$ fadD2::Kan ${ }^{r}$ \\
\hline $\mathrm{SF347}(\Delta \mathrm{fadD} 1-\mathrm{c})$ & SC096 complemented $\Delta$ fadD1 strain; $\mathrm{Gm}^{r}$, $\mathrm{Kan}^{r}$ \\
\hline SF348 ( $\Delta$ fadD2-c) & SC096 complemented $\Delta$ fadD2 strain; $\mathrm{Gm}^{r}, \mathrm{Kan}^{r}$ \\
\hline SF350 & SC096 fadD $1^{\mathrm{T} 214 \mathrm{~A}_{-}-\mathrm{m}^{r}}$ \\
\hline SF351 & SC096 fadD1 ${ }^{\mathrm{G} 216 \mathrm{~A}_{-} \mathrm{Em}^{r}}$ \\
\hline SF352 & SC096 fadD1 ${ }^{\mathrm{T} 217 \mathrm{~A}_{-} \mathrm{Em}^{r}}$ \\
\hline SF353 & SC096 fadD1 ${ }^{\mathrm{G} 219 \mathrm{~A}_{-} \mathrm{Em}^{r}}$ \\
\hline SF354 & SC096 fadD1 ${ }^{\mathrm{E} 362 \mathrm{~A}}-\mathrm{Em}^{r}$ \\
\hline SF355 & SC096 fadD1 G216A $-\mathrm{Em}^{r}, \Delta$ fadD2::Kan ${ }^{r}$ \\
\hline SF356 & SC096 fadD1 G219A-Emr, $\Delta$ fadD2::Kan ${ }^{r}$ \\
\hline
\end{tabular}

Zhang et al., 2012
This study
This study
This study
This study
This study
This study
This study
This study
This study
This study
This study

\section{Plasmids}

\section{pMD19-T (simple) T-vector, Amp ${ }^{r}$}

pK18mobsacB Suicide and narrow-broad-host vector, Kan ${ }^{r}$

pSF115

pET-28b

pBAD24

pBAD24m

p34S-Gm

pSF116

pSF224

pSF225

pSF226

pSF227

pSF228

pSF229

pSF230

pSF231

pSF232

pSF233

pSF234

pSF235

pSF236

pSF237

pSF238

pSF239

pSF240

pSF241

pSF242

pSF243

pSF244

$\mathrm{Kan}^{r}$; expression vector

$\mathrm{Amp}^{r}$; arabinose-inducible vector

fadD1 cloned into pMD19-T; Amp ${ }^{r}$

fadD2 cloned into PMD19-T; Amp ${ }^{r}$ fadD1 in pMD19-T; Ampr ${ }^{r}, \mathrm{Gm}^{r}$ fadD2 in pMD19-T; $\mathrm{Amp}^{r}, \mathrm{Gm}^{r}$ downstream sequences in PMD19-T; Amp ${ }^{r}$
Kanamycin resistance cassette carrying complementation vector; Kan ${ }^{r}$

$\mathrm{Amp}^{r}$; Ncol site of expression vector pBAD24 changed to an Ndel site

Gentamicin resistance cassette carrying vector; $\mathrm{Gm}^{r}$

Gentamicin resistance cassette carrying complementation vector; $\mathrm{Gm}^{r}$

fadD1 from pSF224 inserted between the Ndel and Hindlll sites of pBAD24m; Ampr

fadD2 from pSF224 inserted between the Ndel and Hindlll sites of pBAD24m; Ampr

fadD1 from pSF224 inserted between the Ndel and Hindlll sites of pET28b; Kan ${ }^{r}$

fadD2 from pSF224 inserted between the Ndel and Hindlll sites of pET28b; Kan ${ }^{r}$

A disruption cassette containing gentamicin resistance cassette, the upstream and downstream sequences of

\section{A disruption cassette containing gentamicin resistance cassette, the upstream and downstream sequences of}

PCR-amplified fadD1 cloned between the Kpnl and BamHI sites of pSF116; $\mathrm{Gm}^{r}$

A fragment containing erythromycin resistance cassette and the intact fadD1 gene with upstream and

carrying fadD1 recombination cassette derived from pSF233 with a T214A mutation in FadD1; Em ${ }^{r}$

carrying fadD1 recombination cassette derived from pSF233 with a G216A mutation in FadD1; Em ${ }^{r}$

carrying fadD1 recombination cassette derived from pSF233 with a T217A mutation in FadD1; Em ${ }^{r}$

carrying fadD1 recombination cassette derived from pSF233 with a G219A mutation in FadD1; Em ${ }^{r}$

carrying fadD1 recombination cassette derived from pSF233 with a E362A mutation in FadD1; Em ${ }^{r}$

PCR-amplified fadD2 cloned between the Kpnl and BamHI sites of pSF115, Kan ${ }^{r}$

fadD $1^{\top 214 A}$ from pSF234 inserted between the Ndel and Hindlll sites of pBAD24m; Ampr ${ }^{r}$

fadD1 G216A from pSF235 inserted between the Ndel and Hindlll sites of pBAD24m; Amp ${ }^{r}$

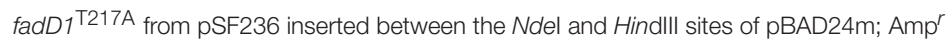

fadD 1 G219A from pSF237 inserted between the Ndel and Hindlll sites of pBAD24m; Amp ${ }^{r}$

fadD ${ }^{\mathrm{E} 362 \mathrm{~A}}$ from pSF238 inserted between the Ndel and Hindlll sites of pBAD24m; Ampr

\section{Takara Inc}

Schafer et al., 1994

Zou et al., 2013

Novagen

Guzman et al., 1995

Zhu et al., 2013

Dennis and Zylstra, 1998

Zhou et al., 2016

This study

This study

This study

This study

This study

This study

This study

This study

This study

This study

This study

This study

This study

This study

This study

This study

This study

This study

This study

This study

This study 
TABLE 2 | Sequences of the PCR primers used in this study.

\begin{tabular}{|c|c|}
\hline Primers & Primer sequences $\left(5^{\prime}-3^{\prime}\right)$ \\
\hline P1 (fadD1-F for T) & CATATGGAAAAAATTTGGTTGA \\
\hline P2 (fadD1-R for T) & AAGCTTTACAATTTTCCTTCCATCT \\
\hline P3 (fadD2-F for T) & CATATGGCTTCTCTCGACTTCCA \\
\hline P4 (fadD2-R for T) & AAGCTTATGAACGTTCATTAACTTAA \\
\hline P5 (fadD1-Uf for mut) & ACCGCTTGTGAGATTGAGTTTAGTTCACA \\
\hline P6 (fadD1-Ur for mut) & $\begin{array}{l}\text { ATGTCAATTCGGGATCCGCGCCACATCTAA } \\
\text { GGTACGTTCA }\end{array}$ \\
\hline P7 (fadD1-Df for mut) & $\begin{array}{l}\text { GATCGGCTTCGTCGACACGTTGTTGCGAGA } \\
\text { TGAGGAAGTT }\end{array}$ \\
\hline P8 (fadD1-Dr for mut) & GTAACTCATAGATCGCATCA \\
\hline P9 (Gm-F) & $\begin{array}{l}\text { CGCGGATCCCGAATTGACATCGAATTGACA } \\
\text { TAAGCCTGTTC }\end{array}$ \\
\hline P10 (Gm-R) & ACGTGTCGACGAAGCCGATCTCGGCTTGAAC \\
\hline P11 (fadD2-Uf for mut) & ACCGCTTGTGATCGGCTTCGTCGACACGT \\
\hline P12 (fadD2-Ur for mut) & $\begin{array}{l}\text { ATGTCAATTCGGGATCCGCGGATCGGCTTC } \\
\text { GTCGACACGT }\end{array}$ \\
\hline P13 (fadD2-Df for mut) & $\begin{array}{l}\text { GATCGGCTTCGTCGACACGTGATCGGCTTC } \\
\text { GTCGACACGT }\end{array}$ \\
\hline P14 (fadD2-Dr for mut) & GATCGGCTTCGTCGACACGT \\
\hline P15 (Kan-F) & $\begin{array}{l}\text { CGCGGATCCCGAATTGACATITTATGGACA } \\
\text { GCAAGCGAA }\end{array}$ \\
\hline P16 (Kan-R) & $\begin{array}{l}\text { ACGTGTCGACGAAGCCGATCTCAGAAGAAC } \\
\text { TCGTCAAGAA }\end{array}$ \\
\hline P17 (fadD1-F for comp) & CGCGTCGACAGTGTTGTGTACTACGGCAG \\
\hline P18 (fadD1-R for comp) & CATGCATGCCTACAATTITCCTTCCATCTGT \\
\hline P19 (fadD2-F for comp) & CGCGTCGACCTTAAGAATACAGACAAACG \\
\hline P2O (fadD2-R for comp) & CATGCATGCTTAAGTTAATGAACGTTCATTA \\
\hline P21 (fadD1-R for PM) & $\begin{array}{l}\text { CTGTGTITATATITICTCGTTCATTACAAT } \\
\text { ПTCCTTCCATCT }\end{array}$ \\
\hline P22 (fadD1-Df for PM) & $\begin{array}{l}\text { AGCTATAAATTATTTAATAAGTAATGAGAATA } \\
\text { AGTGGTATATCT }\end{array}$ \\
\hline P23 (Em-F) & ATGAACGAGAAAAATATAAAACACAG \\
\hline P24 (Em-R) & ТTACTTATTAAATAATTTATAGCT \\
\hline P25 (T214A-F) & $\begin{array}{l}\text { ATCTGGCGTTCTTCAATATGCAGGCGGGA } \\
\text { CAACAGGAGTG }\end{array}$ \\
\hline P26 (T214A-R) & ATATTGAAGAAACGCCAGAT \\
\hline P27 (G216A-F) & $\begin{array}{l}\text { GTTCTTCAATATACAGGCGCAACAACAGGA } \\
\text { GTGGCTAAAGG }\end{array}$ \\
\hline P28 (G216A-R) & CGCCTGTATATTGAAGAAAC \\
\hline P29 (T217A-F) & $\begin{array}{l}\text { TTCTTCAATATACAGGCGGGGCAACAGGAG } \\
\text { TGGCTAAAGGG }\end{array}$ \\
\hline P30 (T217A-R) & CCCGCCTGTATATTGAAGAA \\
\hline P31 (G219A-F) & $\begin{array}{l}\text { ATATACAGGCGGGACAACAGCAGTGGCTAA } \\
\text { AGGGGCAATGC }\end{array}$ \\
\hline P32 (G219A-R) & CTGTTGTCCCGCCTGTATAT \\
\hline P33 (E362A-F) & $\begin{array}{l}\text { TGAAGGTTATGGTATGACCGCATGTTCCCCA } \\
\text { TTGATTGCGG }\end{array}$ \\
\hline P34 (E362A-R) & CGGTCATACCATAACCTTCA \\
\hline P35 (fadD1-R for RT) & AGTTGACCAGCGTACGCT \\
\hline P36 (fadD2-R for RT) & TGACAGACCTGATCAAGT \\
\hline
\end{tabular}

(Continued)
TABLE 2 | Continued

\begin{tabular}{ll}
\hline Primers & Primer sequences $\left(\mathbf{5}^{\prime}\right.$-3') \\
\hline P37 (rp/M-R for RT) & TGCCACCTACATAGCCAG \\
P38 (fadD1-F for RT) & GCGATTGTCGTCGTGTCA \\
P39 (fadD2-F for RT) & TCGTGGACTATTGCTGA \\
P40 (rp/M-F for RT) & GTGACTGGTATGTAGTAG \\
P41 (fadD1-F for PCR test 1) & CCAGTGTTACAGCCAGACA \\
P42 (fadD1-R for PCR test 1) & TAAGATCCGCTTGTGGCGA \\
P43 (fadD2-F for PCR test 2) & GGTGATCGCTTAGACGATCA \\
P44 (fadD2-R for PCR test 2) & AATCATCTTGTTGTGCTAGG \\
P45 (fadD1-F for PCR test 3) & ATTGCCACACGCAGTGAGTT \\
P46 (fadD1-R for PCR test 3) & GGTCATACCATAACCTCAA \\
P47 (fadD2-F for PCR test 3) & GGTGCGACAAATTGCTACTT \\
P48 (fadD2-R for PCR test 3) & TCTTCAGGCTTCTTGTAGTA \\
P49 (fadD1-F for PCR test 4) & ATGGAAAAATTGGTTGA \\
P50 (fadD2-F for PCR test 4) & ATGGCTCTCTCGACTCCA \\
P51 (ompP5d-R for PCR test 4) & AGAATAATTGGTAACAAACCAATA \\
\hline
\end{tabular}

enzymes, to generate the expression vectors. The plasmids were then transformed into the E. coli BL21 strain. The respective FadD1 and FadD2 proteins were expressed at high levels and purified by growing the $\mathrm{FadD}$ expression strains at $37^{\circ} \mathrm{C}$ in $\mathrm{LB}$ medium. When the $\mathrm{OD}_{600}$ reached 0.8 , the cultures were induced by the addition of $0.1 \mathrm{mM}$ isopropyl- $\beta$-D-thio-Dgalactoside (IPTG) and grown at $18^{\circ} \mathrm{C}$ for an additional $16 \mathrm{~h}$ prior to harvest. The cells were centrifuged and collected and then resuspended in lysis buffer containing $50 \mathrm{mM}$ sodium phosphate, $300 \mathrm{mM} \mathrm{NaCl}$, and $10 \mathrm{mM}$ imidazole ( $\mathrm{pH} \mathrm{8.0)}$. The cells were then lysed using ultrasonic disruption and centrifuged to remove unbroken cells and large debris. The supernatants were loaded onto a nickel-ion HisTrap HP affinity column in an ÄKTA Explorer FPLC system (GE). The column was washed with wash buffer $(50 \mathrm{mM}$ sodium phosphate, 300 $\mathrm{mM} \mathrm{NaCl}$, and $40 \mathrm{mM}$ imidazole; $\mathrm{pH} 8.0$ ), and the target proteins were eluted using elution buffer containing $300 \mathrm{mM}$ imidazole. These protein solutions were dialyzed against lysis buffer without imidazole and analyzed using SDS-PAGE and MALDI-TOF.

\section{Measurement of Fatty Acyl-CoA Synthetase Activity}

Fatty acyl-CoA synthetase activity was monitored using Ellman's reagent as previously described (Kang et al., 2010) to detect the amount of free thiol. The reaction buffer mixture contained 150 $\mathrm{mM}$ Tris- $\mathrm{HCl}\left(\mathrm{pH}\right.$ 7.2), $10 \mathrm{mM} \mathrm{MgCl}_{2}, 2 \mathrm{mM}$ EDTA, $0.1 \%$ Triton X-100, $5 \mathrm{mM}$ ATP, $0.5 \mathrm{mM}$ reduced CoA, and fatty acid substrate $(30-300 \mu \mathrm{M})$. The total reaction volume was 450 $\mu \mathrm{l}$ and included $10 \mu \mathrm{g}$ of purified protein. Briefly, to perform the reaction, each mixture containing all of the components listed above (excluding CoA) was assembled, and $405 \mu$ lof the mixture was pre-incubated at $37^{\circ} \mathrm{C}$ for $3 \mathrm{~min}$. The reaction was initiated with the addition of $45 \mu \mathrm{l}$ of $5 \mathrm{mM}$ reduced CoA 
(diluted to a final concentration of $0.5 \mathrm{mM}$ ), which was preincubated at $37^{\circ} \mathrm{C}$ for $3 \mathrm{~min}$, quickly mixed, and incubated at $37^{\circ} \mathrm{C}$ throughout the reaction. Immediately after mixing, a recording was taken at the zero time point by removing $75 \mu \mathrm{l}$ from the $450 \mu \mathrm{l}$ reaction mixture and adding it to $600 \mu \mathrm{l}$ of

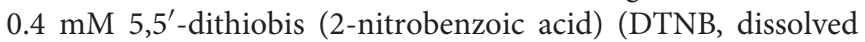
in $0.1 \mathrm{M}$ potassium phosphate at $\mathrm{pH} 8.0$ ), and the absorbance value at $412 \mathrm{~nm}$ was then measured. Subsequently, $75-\mu \mathrm{l}$ aliquots of the reaction mixture were taken at 1-min intervals and mixed with DTNB to obtain additional measurements. The extinction coefficient of CoA was assumed to be $1.36 \times 10^{4} \mathrm{M}^{-1}$ $\mathrm{cm}^{-1}$. All reactions involving FadDs were repeated to obtain triplicate data for each fatty acid at each different concentration. The results were analyzed using Hanes-Woolf plots for various amounts of $[\mathrm{S}]$ against $[\mathrm{S}] / \mathrm{V}$ for each concentration of fatty acid.

\section{Construction of HpfadD Mutants and Complementation Strains}

A suicide plasmid was constructed as follows to disrupt $H$. parasuis fadD1: DNA fragments located approximately $500 \mathrm{bp}$ upstream and 500 bp downstream of $h p f a d D 1$ (D1Up and D1Dn, respectively) were amplified from $H$. parasuis SC096 genomic DNA using the primers P5-P8 (Table 2). The marker gentamicin was amplified from p34S-Gm using the primers P9 and P10. All of the fragments were connected using overlap PCR with primers P5 and P8 and cloned into PMD19-T to obtain the plasmid pSF230, which carried the fadD1 disruption cassette. The fadD2 upstream and downstream fragments were amplified using the same method, except that the primers P11-P14 were used. The $\mathrm{Kan}^{r}$ gene was amplified from pK18mobsacB using the primers p15 and P16. These primers were ligated using overlap PCR with the primers P11 and P14 to obtain the plasmid pSF231, which carried a fadD2 disruption cassette. These plasmids were transformed into SC096 to generate the fadD1 or fadD2 mutants SF345 and SF346, respectively, via natural transformation, as previously described (Zhang et al., 2012). The fadD1 complementation strain was constructed as follows: an intact $f a d D 1$ fragment with its native promoter was amplified with primers P17 and P18. The PCR product of the fadD1 fragment was purified and digested with KpnI and BamHI. The fragment was then cloned into the complementation vector pSF115 (Zou et al., 2013) that was digested using the same enzymes to obtain the plasmid pSF232. The pSF232 plasmid was then transformed into SF345 ( $f a d D 1$ mutant) to generate the fadD1 complementation strain SF347. A fadD2 complementation plasmid was constructed using a similar method with the primers P19 and P20 and the complementation vector pSF116 (Zhou et al., 2016). The fadD2 plasmid was transformed into SF346 (fadD2 mutant) to obtain the fadD2 complementation strain SF348.

\section{Site-Directed Mutagenesis and Essentiality Testing}

To obtain a fadD1 site-directed mutation strain, a mutation plasmid was constructed as follows: a fragment containing D1Up and intact fadD1 was amplified using SC096 genomic DNA and the primers P5 and P21. Another fragment containing the intact fadD1 gene and D1Dn was amplified using the primers P22 and P8. The erythromycin resistance gene $\mathrm{Em}^{r}$ was amplified using the primers P23 and P24. The fragments were connected using overlap PCR with the primers P5 and P8. The resulting PCR product was cloned into pMD19-T to obtain the template plasmid pSF233, which carried an intact fadD1 gene containing upstream and downstream $\mathrm{Em}^{r}$ inserts at the end of $\mathrm{fadD} 1$. Five point mutations were generated using the primers P25P34. For example, to place the T214A mutation in FadD1, an upstream fragment was amplified using the P5 primer and T214A point mutation primer P25 and using PSF233 as a template. The downstream fragment was amplified using the same template with primers P26 and P8. The two fragments were ligated using overlap PCR and cloned into pMD19T to obtain the plasmid pSF234, which carried the fadD1 recombination cassette containing the T214A mutation in FadD1. The mutation vector was then transformed into SF345 (fadD1 mutant) to generate the fadD $1^{\mathrm{T} 214 \mathrm{~A}}$ mutation strain SF350. The other four sitedirected mutation strains (G216A, T217A, G219A, and E362A) were constructed using a similar method. To further confirm the essentiality of the fadD genes, fadD2 disruption strains were constructed by natural transformation using fadD1 site-directed mutation strains as recipients. The inability to obtain a mutation indicated that the selected mutation sites in FadD1 were essential for FadD1 activity and for supporting bacteria survival in a fadD2 mutant.

\section{Analysis of Fatty Acid Composition}

A cellular lipid assay was performed as previously described (Zhu et al., 2013) with some modifications. Briefly, the $H$. parasuis strains were grown to mid-logarithmic phase in TSB medium containing bovine serum and NAD. The cells were harvested and washed three times with water. The cells were resuspended in $0.8 \mathrm{ml}$ of water, and $1 \mathrm{ml}$ chloroform and $2 \mathrm{ml}$ methanol were then added. An additional $1 \mathrm{ml}$ of water and $1 \mathrm{ml}$ of chloroform were added after the solution was shaken for $1 \mathrm{~h}$ at room temperature. The suspensions were vortexed for $10 \mathrm{~s}$ and then centrifuged. The lower organic phase was extracted and washed twice with $2 \mathrm{M} \mathrm{KCl}$ and once with water and then dried in nitrogen. The phospholipids were dissolved in $1.2 \mathrm{ml}$ of dry methanol, and $0.2 \mathrm{ml}$ of $25 \%$ ( $\mathrm{vol} / \mathrm{vol}$ ) sodium methoxide (Sigma) was added. A 1.2-ml volume of $2 \mathrm{M} \mathrm{HCl}$ was added after the solution was incubated for $30 \mathrm{~min}$ at room temperature. The fatty acid methyl esters were extracted using petroleum ether. The extraction agent was removed using a stream of nitrogen, and the samples were analyzed using gas chromatography (GC)-mass spectrometry in an Agilent 7890-5975C. The GC was equipped with a (5\%-phenyl)-methyl polysiloxane HP-5MS column (60 $\times 250 \times 0.25 \mu \mathrm{m})$. A chromatogram was produced by initially holding the oven temperature at $110^{\circ} \mathrm{C}$ and then increasing the temperature to $140^{\circ} \mathrm{C}$ at a rate of $5^{\circ} \mathrm{C} / \mathrm{min}$. The temperature was then held for $5 \mathrm{~min}$, increased at a rate of $5^{\circ} \mathrm{C} / \mathrm{min}$ to $180^{\circ} \mathrm{C}$, and held for $5 \mathrm{~min}$ at $180^{\circ} \mathrm{C}$. The temperature was increased to $250^{\circ} \mathrm{C}$ at a rate of $5^{\circ} \mathrm{C} / \mathrm{min}$ and held for $5 \mathrm{~min}$. Finally, the temperature was increased to $280^{\circ} \mathrm{C}$ at a rate of $5^{\circ} \mathrm{C} / \mathrm{min}$ and held at $280^{\circ} \mathrm{C}$ 
A
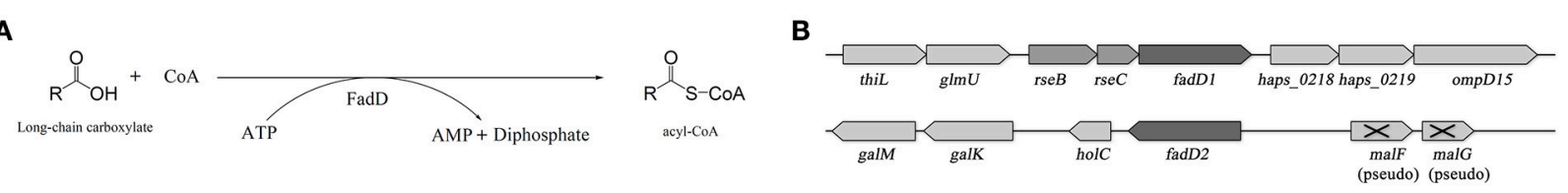

C Escherichia coli MG1655 FadD
Haemophilus influenzae Rd KW20 FadD
Heamohilus parasusis SH0165 Fadd1
Heemophil lus parasuis SH0165 FadD2

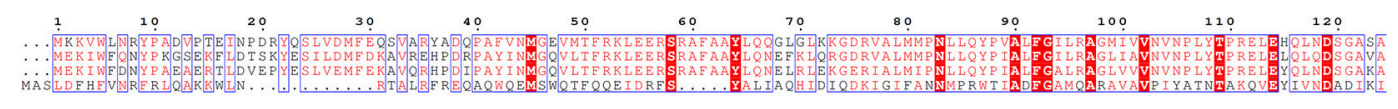

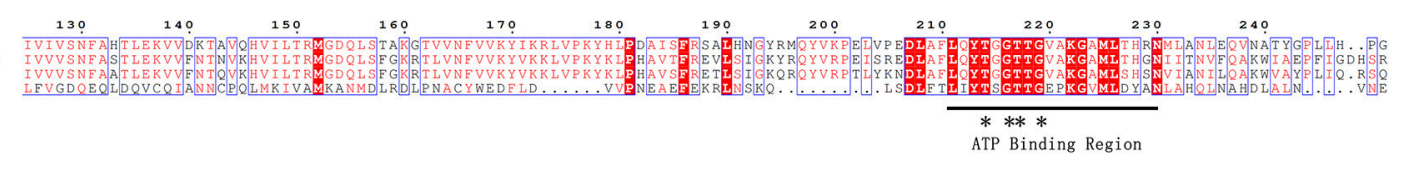

Haemophilus influenzae Rd KH2O Fad Haemophil us influenzac Rd KK20 Fad
Haemohilus parasuis SHO165 FadD1
Heemophi lus parasuis SHO165 FadD2

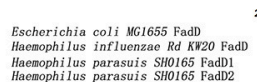
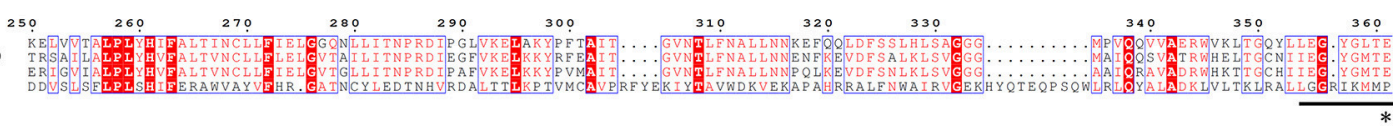

Escherichia coli MG1655 FadD
Haemophil us influenzae Rd KH20 FadD

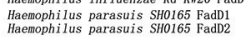
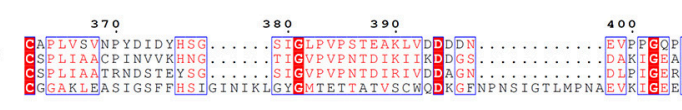

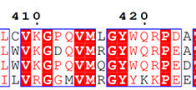

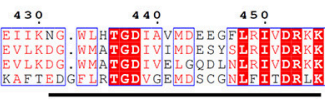

FCL motif
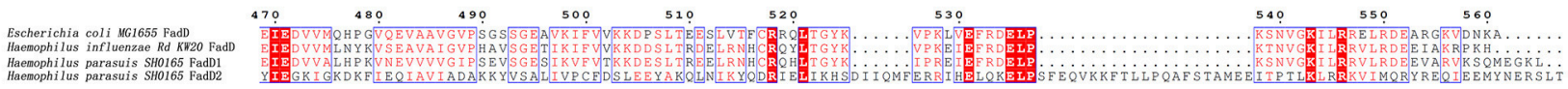

FIGURE 1 | The acyl-CoA synthetase (FACS) reaction, organization of the fadD genes clusters, and alignment of $\boldsymbol{H}$. parasuis FadDs with homolog of E. coli and $\boldsymbol{H}$. influenza. (A) The chemical equation of acyl-CoA synthetase reaction. (B) Organization of $H$. parasuis fadDs clusters. The filled arrows indicate the relative size and transcriptional direction of the genes. The numbers and names below the arrows indicate the gene annotations in the $\mathrm{H}$. parasuis SH0165. The res $B$ and res $C$ genes encode cytochrome $\mathrm{C}$ biosynthesis-related proteins. The gene holC encodes DNA polymerase III chi subunit. (C) Alignment of $H$. parasuis FadDs with homologous of E. coli and H. influenza. The ATP/AMP and FACS motifs are indicated by underlines. Sites of experiment confirmed in E. coli are denoted by asterisks.

for $5 \mathrm{~min}$. The following conditions were used for mass detector: capillary direct interface temperature, $260^{\circ} \mathrm{C}$; ionization energy, $70 \mathrm{eV}$; and mass range, $35-550 \mathrm{amu}$. Helium was used as the carrier gas (flow rate, $1.5 \mathrm{ml} / \mathrm{min}$ ). The components in essential oils were identified using the NIST11 MS data library.

\section{RNA Extraction and Quantitative Real-Time PCR Analyses}

The $H$. parasuis strains were cultured to mid-logarithmic phase in TSB medium containing heat-inactivated newborn bovine serum and NAD. Total RNA was extracted using a Bacterial RNA Isolation Kit (Omega). The RNA was adjusted to a concentration of 200-500 ng/ $\mu \mathrm{l}$ (measured using a NanoDrop 8000, Thermo Scientific), and all samples were reverse-transcribed using a PrimeScript ${ }^{\mathrm{TM}}$ RT Reagent Kit (Takara) with the specific primers P35, P36, and P37. Quantitative real-time PCR analyses were performed using an Applied Biosystems PRISM model 7500 Sequence Detection system with SYBR ${ }^{\circledR}$ Premix Ex Taq ${ }^{\mathrm{TM}}$ (Takara). The results were relatively quantified using the comparative cycle threshold method. The endogenous internal control $r p l M$ was used for sample normalization, as previously described (Zhou et al., 2016). The amplification program was as follows: $30 \mathrm{~s}$ at $95^{\circ} \mathrm{C}$ followed by 40 cycles at $95^{\circ} \mathrm{C}$ for $5 \mathrm{~s}$ and $60^{\circ} \mathrm{C}$ for $34 \mathrm{~s}$. Three independent experiments were performed for each strain, and three technical replicate RT-PCRs were performed for each sample.

\section{Serum Bactericidal Assay}

The serum bactericidal assays were performed using porcine serum as previously described (Zou et al., 2013). Porcine serum stored at $-80^{\circ} \mathrm{C}$ from four healthy piglets (3-4 weeks old) from a farm free of Glässer's disease collected in previous work (Zhou et al., 2016) was used for this study. The piglets were free of Glässer's disease. The serum was filter-sterilized $(0.22 \mu \mathrm{M})$, and aliquots were stored at $-80^{\circ} \mathrm{C}$. The controls were performed using serum samples that were heated at $56^{\circ} \mathrm{C}$ for $30 \mathrm{~min}$. Wildtype and recombinant $H$. parasuis strains were grown to midlogarithmic phase. A 100 or $400-\mu l$ volume of fresh porcine serum or heat-treated serum was mixed with $100 \mu \mathrm{l}$ of a diluted bacterial suspension $\left(10^{8} \mathrm{CFU}\right)$ to achieve a final concentration of 50 or $80 \%$ serum. The mixture was then incubated at $37^{\circ} \mathrm{C}$ for $1 \mathrm{~h}$. The bacteria were serially diluted and plated on TSA plates containing heat-inactivated newborn bovine serum and NAD. To quantify the results, the plates were incubated for $24 \mathrm{~h}$ at $37^{\circ} \mathrm{C}$ in an atmosphere containing $5 \% \mathrm{CO}_{2}$. The percent survival was calculated as the ratio of the number of colonies grown from fresh serum to the number grown from heat-treated serum. Three independent experiments were performed for each $H$. parasuis strain. 


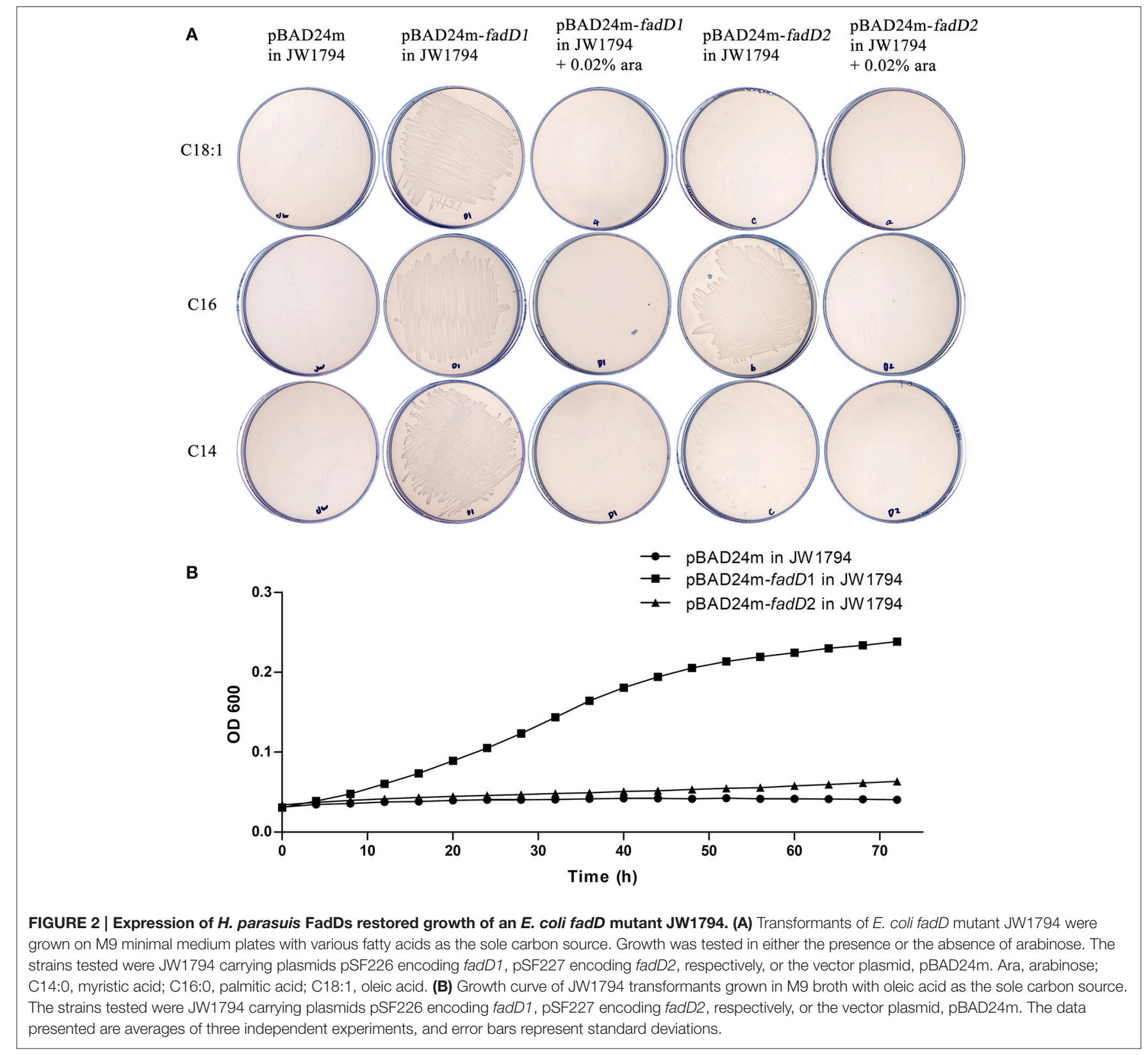

\section{Antimicrobial Susceptibility Testing}

To control the inoculum volume, the antimicrobial susceptibility of all of the $H$. parasuis strains was determined using the agar dilution method with an antibacterial determiner (SAKUMA, Japan). The results were interpreted according to the Clinical and Laboratory Standards Institute (CLSI) guidelines. The MIC value was defined as the lowest concentration that resulted in no visible colonies. The reference strains Actinobacillus pleuropneumoniae ATCC 27090 and Escherichia coli ATCC 25922 were used as quality controls for the MIC determination.

\section{Statistical Analysis}

Student's $t$-test was used to determine the significance of differences. A $p$-value $<0.05$ was considered statistically significant.

\section{RESULTS}

\section{Two Predicted Acyl-CoA Synthetase Genes in $\boldsymbol{H}$. parasuis}

In this study, two $H$. parasuis genes, fadD1 (HAPS_0217) and fadD2 (HAPS_1695), were annotated and found to encode homolog of E. coli FadD, which is a FACS. FACSs belong to the ANL superfamily of adenylating enzymes. Members of this family catalyze two partial reactions: the first is the adenylation of a carboxylate to form an acylAMP intermediate, which is followed by a second reaction that usually leads to the formation of a thioester (Gulick, 2009). The reaction product of FadD is acyl-CoA (Figure 1A). A sequence comparison of members of the FACS protein family revealed the presence of an ATP-AMP signature 

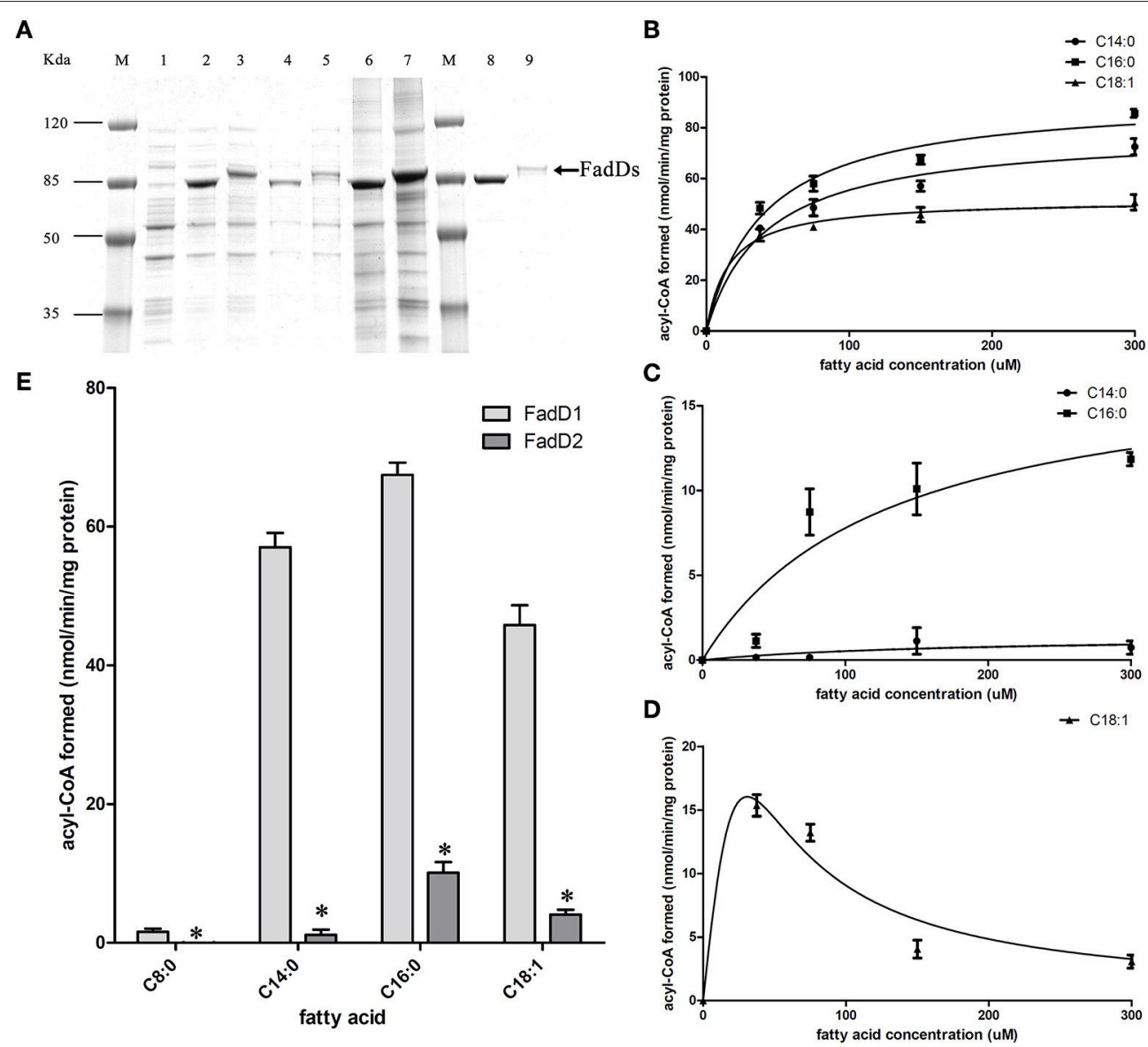

FIGURE 3 | Purification and biochemical characterization of the two $\boldsymbol{H}$. parasuis FadDs. (A) Purification of $H$. parasuis SC096 FadD1 and FadD2 by native nickel-chelate chromatography. Lane M, molecular mass markers; lane 1-3, proteins from whole cells extraction of BL21 with the vector plasmid pET28b, FadD1, and FadD2 expression strains; lane 4 and 5, supernatant of lysate from FadD1 or FadD2 expression strain; lane 6 and 7 , centrifuged precipitation of lysate from FadD1 or FadD2 expression strain; lane 8, purified FadD1; lane 9, purified FadD2. (B,C) Kinetics of FadD1 and FadD2 activity was determined using fatty acids of different concentrations (30-300 $\mu \mathrm{M}$ ) as the variable substrates. (D) Kinetics of FadD2 inhibition with oleic acid was determined using variable concentrations (30-300 $\mu \mathrm{M})$ of oleic acid as the fatty acid substrates. (E) Activities comparison of FadD1 and FadD2 were performed using $150 \mu \mathrm{M}$ oleic acid as a fatty acid substrate. The error bars of all the experiments represent the mean \pm S.D. $(n=4)$. The asterisks indicated that activity of FadD1 was statistically different $(P<0.01)$ from that of the FadD2 as judged by the Student's $t$-test.

motif and a FACSs conserved motif (Black and DiRusso, 2003).

$H p f a d D 1$ is located in a chromosomal cluster with resB and res $C$ (a putative cytochrome c biogenesis-associated protein), whereas HpfadD2 is located at another locus and is likely to be monocistronic (Figure 1B). The gene cluster structures of both genes shared no similarity with the E. coli fadD locus. An alignment of the FadD homolog revealed that HpFadD1 was $65 \%$ identical to E. coli FadD and that the ATP/AMP and FACS motifs were conserved between them (Figure 1C). In contrast, HpFadD2 shared no similarity with EcFadD when analyzed using BLAST but was $22 \%$ identical to E. coli FadK, a short-chain acylCoA synthetase (Morgan-Kiss and Cronan, 2004). Interestingly, HpFadD2 was 25\% identical to HpFadD1 and contained an ATP/AMP motif. This analysis indicates that FadD1 and FadD2 may have acyl-CoA synthetase activity.

\section{$H$. parasuis fadD1 and fadD2 Functionally Replace E. coli fadD In vivo}

We used an E. coli complementation system to determine whether FadD1 or FadD2 functions as an acyl-CoA synthetase. The E. coli JW1794 strain is a fadD mutant that cannot survive in minimal medium when exogenous fatty acid is the sole source of carbon (Baba et al., 2006). This deficiency in fadD can be restored by a plasmid carrying the gene encoding acylCoA synthetase. Hence, fadD1 and fadD2 were cloned into the arabinose-inducible vector $\mathrm{PBAD} 24 \mathrm{~m}$ to produce the expression constructs pSF226 and pSF227, respectively. These plasmids were then transformed into the E. coli strain JW1794. The resulting transformants were tested on M9 medium plates supplemented with different fatty acids as the sole carbon source.

An examination of the growth of the complementation strains incubated on M9 minimal medium plates revealed that the 
TABLE 3 | Kinetic properties of FadD1 and FadD2 with various substrates.

\begin{tabular}{lccccc}
\hline FACS & Substrates & \multicolumn{4}{c}{ Kinetic parameters $^{\boldsymbol{a}}$} \\
\cline { 3 - 6 } & & $\boldsymbol{V}_{\mathbf{m a x}}{ }^{\boldsymbol{b}}$ & $\boldsymbol{K}^{\boldsymbol{c}}$ & $\boldsymbol{k}_{\text {cat }}{ }^{\boldsymbol{d}}$ & $\boldsymbol{k}_{\text {cat } /} \boldsymbol{K m}^{\boldsymbol{e}}$ \\
\hline FadD1 & $\mathrm{C} 14: 0$ & 78.42 & 41.48 & 0.083 & 2.01 \\
& $\mathrm{C} 16: 0$ & 92.11 & 39.90 & 0.098 & 2.45 \\
& $\mathrm{C} 18: 1$ & 51.64 & 15.64 & 0.055 & 3.51 \\
\hline FadD2 & $\mathrm{C} 14: 0$ & 1.45 & 173.40 & 0.002 & 0.01 \\
& $\mathrm{C} 16: 0$ & 17.78 & 128.30 & 0.020 & 0.16 \\
& $\mathrm{C} 18: 1$ & $\mathrm{ND}$ & $\mathrm{ND}$ & $\mathrm{ND}$ & $\mathrm{ND}$ \\
\hline
\end{tabular}

${ }^{a}$ Kinetic constants $\left(K_{m}\right.$ and $\left.V_{\max }\right)$ defined using the Hanes-Woolf plot.

${ }^{b}$ Nanomole of acyl-COA formed/min/mg of protein.

${ }^{c} \mu M$ of FA.

$d_{s^{-1}}$; determined using MW of FadD1(63684) and FadD2 (68500).

${ }^{e} \mathrm{mM}^{-1} \mathrm{~s}^{-1}$.

JW1794 strain carrying fadD1 grew on various fatty acids, whereas JW1794 carrying fadD2 grew only on C16:0 (Figure 2A). These strains grew only in the absence of the inducer arabinose, indicating that high levels of expression of either FadD may be toxic, consistent with previous findings (Bi et al., 2014). To confirm that this phenotype was consistent when these strains were grown in M9 broth, we determined the growth curves of these strains. We found that the HpfadD2 complementation strain survived when grown on minimal medium in which oleic acid was the sole carbon source, although it grew quite slowly compared with the HpfadD1 complementation strain (Figure 2B, the doubling times of the HpfadD1 and HpfadD2 complementation strains were $13.38 \pm 2.16 \mathrm{~h}$ and $37.36 \pm$ 12.51 h, respectively, $P<0.05)$. Altogether, these results indicate that FadD1 and FadD2 have acyl-CoA synthetase activity in vivo.

\section{Purification and Characterization of the FadD1 and FadD2 Proteins}

We produced His-tagged proteins to confirm the substrates of the two FACSs. FadD1 and FadD2 were expressed at high levels in $E$. coli grown in low-temperature induction conditions. The expression levels of the two enzymes were equivalent in whole cell protein extracts, but after ultrasonication and centrifugation, the lysed supernatant of the FadD2-expressing strain contained less of the target protein. Non-transparent inclusion bodies were not observed, indicating that FadD2 may be localized mainly in particulate fractions, as described in previous investigations that showed that EcFadD was located in both the soluble and particulate fractions of bacterial cells (Kameda and Nunn, 1981). The proteins were purified using nickel chelate chromatography in an AKTA explorer FPLC system. Target proteins were detected using SDS-PAGE (Figure 3A) and confirmed by mass analysis (Figure S1).

The fatty acid substrate specificity of each FadD was determined in vitro. FadD1 exhibited a high level of activity on C14:0, C16:0, and C18:1, with high $V_{\max }$ (78.42, 92.11, and 51.64 nanomoles of acyl-CoA formed $/ \mathrm{min} / \mathrm{mg}$ of protein, respectively) and low $K_{\mathrm{m}}$ values $(41.48,39.90$, and $15.64 \mu \mathrm{M}$, respectively) when the substrate ranged from 30 to $300 \mu \mathrm{M}$ (Figure 3B and Table 3). FadD2 showed clear activity only on
C16:0 (17.78 nanomoles of acyl-CoA formed $/ \mathrm{min} / \mathrm{mg}$ of protein for $V_{\max }, 128.30 \mu \mathrm{M}$ for $\left.K_{\mathrm{m}}\right)$, and its level of activity was very low on C14:0 (1.45 nanomoles of acyl-CoA formed/min/mg of protein for $V_{\max }, 173.40 \mu \mathrm{M}$ for $K_{\mathrm{m}}$ ) (Figure 3C and Table 3). Moreover, oleic acid in different concentrations had distinct substrate inhibition effects on FadD2. A higher concentration of C18:1 resulted in lower FadD2 activity (Figure 3D). This may explain why the JW1794 strain carrying FadD2 could not grow on C18:1 (Figure 2A). The fact that FadD1 had higher $k_{\text {cat }} K_{\mathrm{m}}$ values on all selected FAs indicated that FadD1 had a higher level of activity than FadD2 (Table 3). These results are in accordance with the E. coli complementation testing results (Figure 2). FadD1 and FadD2 are long-chain FACS, as they both have higher LCFA (long-chain fatty acid) and MCFA (mediumchain fatty acid) activity than SCFA (short-chain fatty acid, such as C8:0) activity (Figure 3E). Therefore, FadD2 is not a shortchain FACS as FadK is in E. coli, although they share some homology.

\section{Either fadD1 or fadD2 Is Essential for H. parasuis SC096 Survival}

To determine the physiological functions of the two FACS in H. parasuis, fadD1, or fadD2 mutants were constructed using natural transformation, as described in the methods section (Figure 4). However, double mutants were not produced when the fadD1 disruption vector was transformed into the fadD2 mutant or when the fadD2 disruption vector was transformed into the fadD1 mutant. These transformation experiments were performed more than five times each; however, no positive colonies were obtained. Notably, the complementation strains of $\Delta f a d D 1$ or $\Delta f a d D 2$ were obtained as described in the methods section (Figure 4 and Figure S2). These results indicated that the natural transformation ability of the $\Delta f a d D 1$ and $\Delta f a d D 2$ strains was not significantly attenuated.

In a previous study, residue substitutions were introduced in the ATP/AMP signature motif of E. coli FadD to identify the specific residues that are critical for its activity (Weimar et al., 2002). To further determine whether fadD1 or fadD2 is essential for $H$. parasuis survival, we performed genomic site-directed mutagenesis of five conserved amino acids in the ATP/AMP motif of FadD1. Schematics for the constructed strains are shown in Figure 4A. All of the strains were confirmed by PCR and sequencing (Figure 4B). First, the fadD $1^{\mathrm{T} 214 \mathrm{~A}}$, $f a d D 1^{\mathrm{G} 216 \mathrm{~A}}, f a d D 1^{\mathrm{T} 217 \mathrm{~A}}, f a d D 1^{\mathrm{G} 219 \mathrm{~A}}$, and $f a d D 1^{\mathrm{E} 362 \mathrm{~A}}$ strains were constructed. Subsequently, all site-directed mutants were naturally transformed with pSF231 (a fadD2 disruption vector) to generate double-mutant strains. However, only fadD1 ${ }^{\mathrm{G} 216 \mathrm{~A}}$ $\triangle$ fadD2 and $f a d D 1^{\mathrm{G} 219 \mathrm{~A}}-\Delta f a d D 2$ were obtained (Table 4). Moreover, the growth of E. coli JW1794 cells carrying plasmids encoding the mutant proteins (genes with point mutations contained in the expression vector pBAD24m) was also tested on oleate (Figure 4C). This assay demonstrated that FadD1 G216A or FadD $1^{\mathrm{G} 219 \mathrm{~A}}$ exhibited activity that was sufficient to support a similar level growth as that of JW1794 carrying wild-type FadD1 on minimal medium when oleic acid was the sole carbon source. Growth was summarized for of all of the complementation 
A

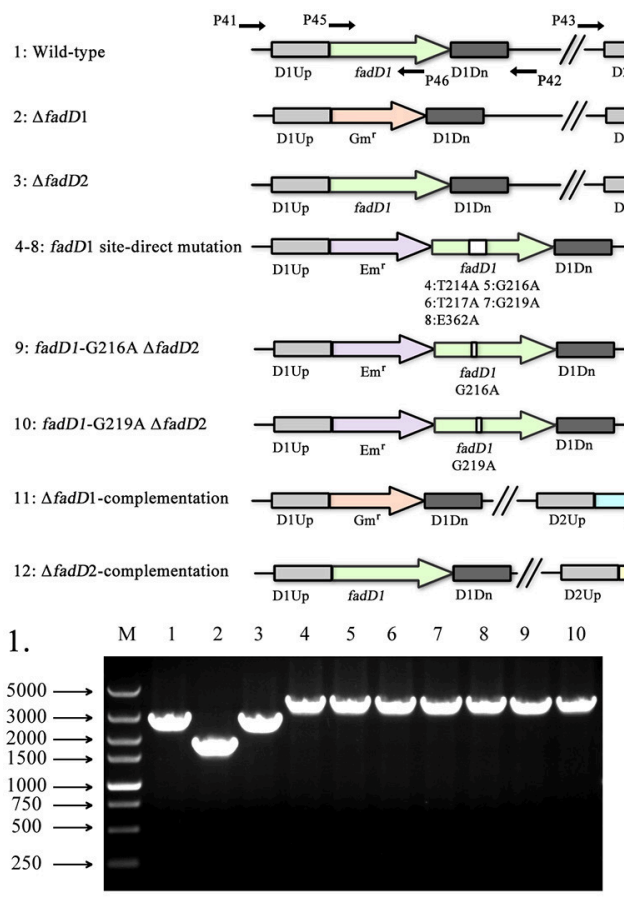

3.
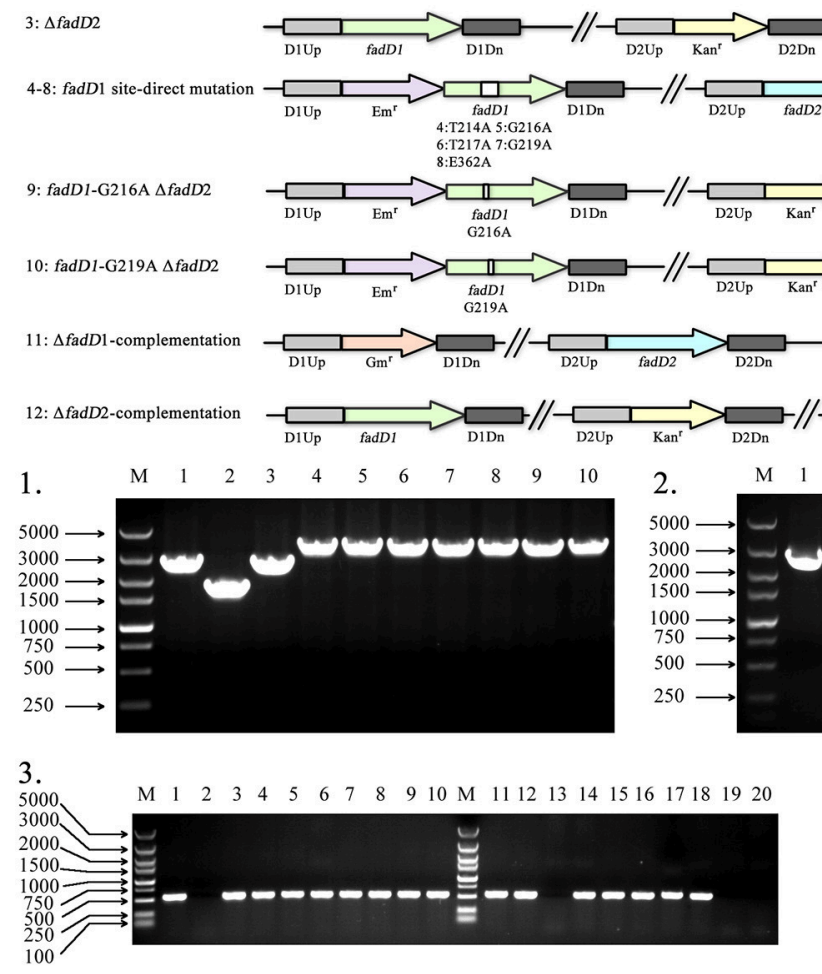
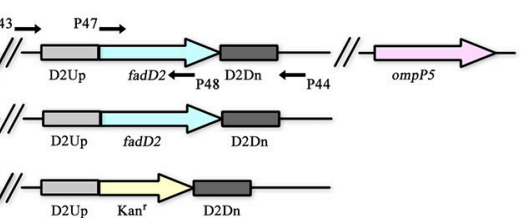

$$
\text { . }
$$$$
{ }_{\mathrm{D} 2 \mathrm{Up}} \mathrm{fadD2}
$$$$
\text { Cᄂ }
$$$$
/ /-\underset{\text { D2Up }{ }_{\mathrm{Kan}^{\mathrm{r}}}}{\longrightarrow}
$$
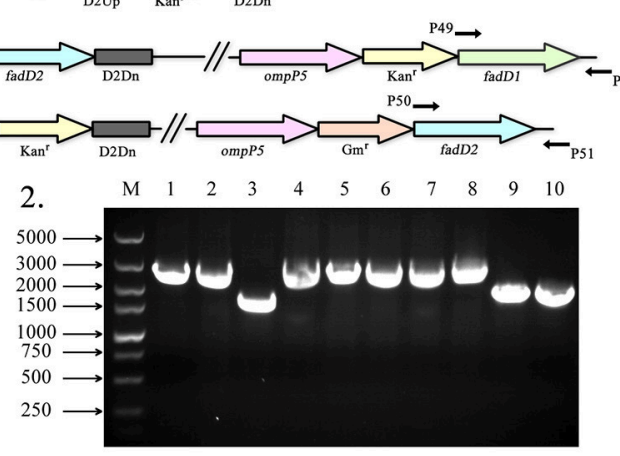

4.

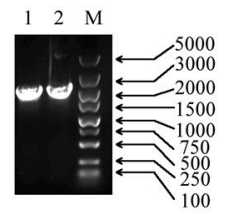

c
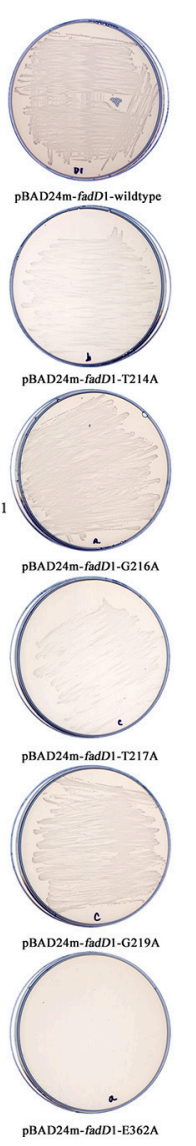

FIGURE 4 | Construction of in-frame deletion and site-direct mutants of fadD genes in $\boldsymbol{H}$. parasuis. (A) Schematic diagram of mutants constructed in this study. Kan ${ }^{r}$, kanamycin-resistant gene; $\mathrm{Gm}^{r}$, gentamicin-resistant gene; D1Up, upstream sequence of fadD1; D1Dn, downstream sequence of fadD1; D2Up, upstream sequence of fadD2; D2Dn, downstream sequence of fadD2; T214A, threonine of the site 214 substituted to alanine in FadD1; G216A, glycine of the site 216 substituted to alanine in FadD1; T217A, threonine of the site 217 substituted to alanine in FadD1; G219A, glycine of the site 219 substituted to alanine in FadD1; E362A, glutamic acid of the site 362 substituted to alanine in FadD1. (B) Identification of mutations by PCR. Part 1 showed the PCR analysis of fadD1 region in all 10 strains using primers P41 and P42 as shown in panel A (lane 1, SC096; lane 2, $\Delta$ fadD1; lane 3, $\Delta$ fadD2; lane 4, fadD1 T214A; lane 5, fadD1G216A; lane 6, fadD1 ${ }^{\mathrm{T} 217 \mathrm{~A}}$; lane 7, fadD1 G219A; lane 8, fadD1 E362A; lane 9, fadD1G216A $-\triangle$ fadD2; lane 10, fadD1 G219A $-\triangle$ fadD2). Part 2 showed the PCR analysis of fadD2 region using primers P43 and P44 (lane 1, SC096; lane 2, $\Delta f a d D 1$; lane 3, $\Delta$ fadD2; lane 4, fadD 1 ${ }^{\mathrm{T} 214 \mathrm{~A}}$; lane 5, fadD1 G216A; lane 6, fadD 1 T217A; lane 7, fadD1 G219A; lane 8, fadD1 $\mathrm{E} 362 \mathrm{~A}$; lane 9, fadD1 $\mathrm{G} 216 \mathrm{~A}-\triangle \mathrm{fadD2}$; lane 10, fadD1G219A $-\Delta f a d D 2)$. The in-frame region of fadD1 and fadD2 were detected using primers $\mathrm{P} 45-\mathrm{P} 48$ as shown in Part 3 (lane 1, SC096; lane 2, $\Delta$ fadD1; lane 3, $\Delta$ fadD2; lane 4, fadD1 T214A; lane 5, fadD1G216A; lane 6, fadD1 T217A; lane 7, fadD1 G219A; lane 8, fadD1 E362A; lane 9, fadD1G216A $-\Delta f a d D 2$; lane 10, fadD1G219A $-\Delta$ fadD2; lane 11, SC096; lane 12, $\Delta$ fadD1; lane 13, $\Delta$ fadD2; lane 14, fadD1T214A; lane 15, fadD1 G216A; lane 16, fadD1 T217A; lane 17, fadD1 G219A; lane 18, fadD1 E362A; lane 19, fadD1G216A $-\triangle$ fadD2; lane 20, fadD1G219A $-\triangle$ fadD2). Part 4 confirmed the complementation strain using primers P49-P51 as shown in panel A (lane 1, $\Delta$ fadD1-complementation and lane 2, $\Delta$ fadD2-complementation). (C) Transformants of E. coli fadD mutant JW1794 were grown on M9 minimal medium plates with oleic acid as the sole carbon source. The strains tested were JW1794 carrying plasmids pSF226, pSF240, pSF241, pSF242, pSF243, or pSF244 encoding fadD1, fadD1 ${ }^{\mathrm{T} 214 \mathrm{~A}}$, fadD1G216A , fadD1 ${ }^{\mathrm{T} 217 \mathrm{~A}}$, fadD ${ }^{\mathrm{G} 219 \mathrm{~A}}$, or fadD1 ${ }^{\mathrm{E} 362 \mathrm{~A}}$, respectively.

strains derived from the E. coli JW1794 fadD mutant in Table 5. In conclusion, these data indicated that a fadD2 mutant can be generated only when the strain has sufficient FadD1 activity. Hence, either fadD1 or fadD2 is required for the survival of this bacterium.

\section{The Effect of fadD Gene Deletion on Growth and Serum Resistance}

Next, the fadD1 and fadD2 mutants were tested to determine their physiological and virulence phenotypes. We tested the growth of the wild-type strain and the mutants in TSB medium containing bovine serum and NAD. Only negligible changes in growth rates were detected in cells grown in this rich medium (Figure 5A). An RT-PCR analysis of total $H$. parasuis RNA extracted from log-phase cells showed that the expression level of fadD2 was not significantly altered in the fadD1 mutant (Figure 5B, the relative expression levels of fadD1 in WT and $\triangle$ fadD2 were $0.71 \pm 0.14$ and $0.66 \pm 0.10$, respectively, $P>$ 0.05). Similarly, the level of fadD1 transcription in the $\triangle \mathrm{fadD} 2$ strain was equivalent to that in the wild-type strain (Figure 5C, the relative expression levels of fadD2 in WT and $\Delta f a d D 1$ 
TABLE 4 | Construction of fadD mutants in this study.

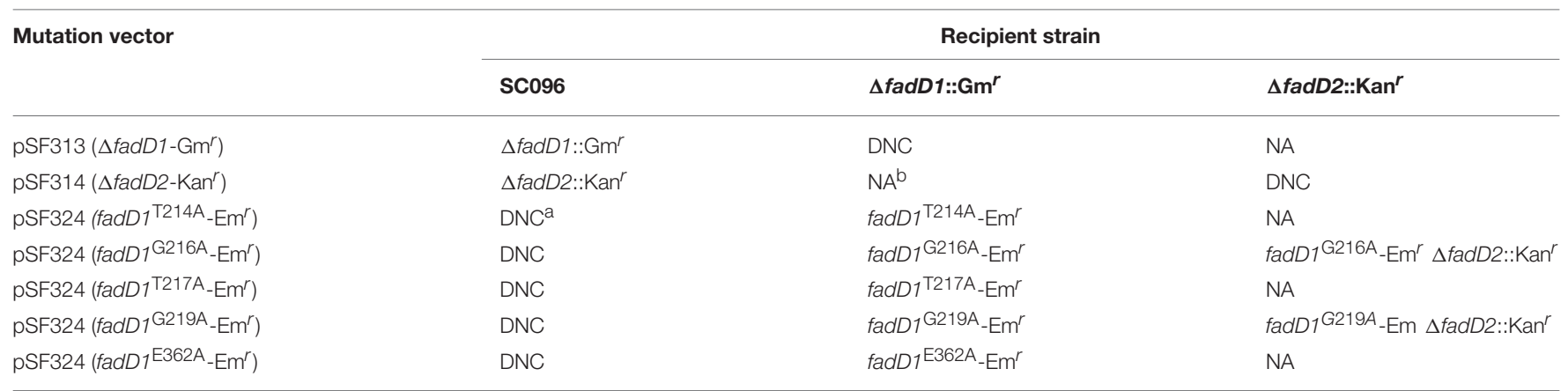

${ }^{a}$ DNC, Did not construct.

${ }^{b}$ NA, Not available.

TABLE 5 | Growth of $E$. coli complementation strains on fatty acids of varying chain length.

\begin{tabular}{|c|c|c|c|c|c|c|c|c|c|}
\hline C-source & \multicolumn{9}{|c|}{ Strains } \\
\hline C14:0 & - & - & + & + & $N D^{a}$ & ND & ND & ND & ND \\
\hline C16:0 & - & - & + & + & ND & ND & ND & ND & ND \\
\hline
\end{tabular}

${ }^{a} N D$, Not determined.

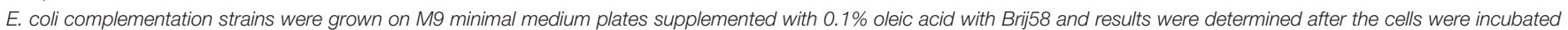
for 72 or $120 \mathrm{~h}$ at $37^{\circ} \mathrm{C}$. Growth was denoted as plus sign if growth after $72 \mathrm{~h}$ was observed or minus sign when no colonies were apparent at $120 \mathrm{~h}$ of incubation.

were $5.81 \pm 0.58$ and $5.31 \pm 0.61$, respectively, $P>0.05)$. When growth testing was performed using a chemically defined medium (CDM) containing oleic acid, the generation time for the fadD1 mutant was appreciably longer than that for the wildtype strain SC096 and its complementation strain (Figure 5D, the doubling times of SC096 and the $\Delta f a d D 1$ and $\Delta f a d D 1$ complementation strains were $3.31 \pm 0.17,4.98 \pm 0.07$, and $3.93 \pm 0.25 \mathrm{~h}$, respectively, $P<0.05)$. Smaller colonies of the fadD1 mutant grew on CDM agar, and these exhibited a phenotype consistent with the broth medium testing results. Additionally, no visible growth was detected when the wild-type strain was grown on CDM agar without fatty acids, indicating that acyl-CoA may be indispensable for the survival of $H$. parasuis (Figure 5E). Furthermore, the roles of the fadD genes in $H$. parasuis serum resistance were investigated. However, there was no significant difference in sensitivity between the wildtype strain and its mutants (Figure 5F, the survival rates of SC096, $\Delta f a d D 1$ and $\Delta f a d D 2$ in $50 \%$ porcine serum were 71.6 $\pm 6.6,69.6 \pm 4.9$, and $71.9 \pm 3.4 \%$, respectively. The survival rates of SC096, $\Delta f a d D 1$ and $\triangle f a d D 2$ in $80 \%$ porcine serum were $23.6 \pm 5.7,24.5 \pm 3.7$, and $19.9 \pm 5.1 \%$, respectively, $P>0.05)$.

\section{FadD1 Plays a Role in $\boldsymbol{H}$. parasuis Quinolone Antibiotic Resistance}

A summary of the MIC values of several antibiotics that were evaluated in this study is shown in Table 6. Interestingly, the fadD1 mutant was more susceptible to all of the selected quinolone antibiotics, including levofloxacin, enrofloxacin, nalidixic acid, and ciprofloxacin, whereas the fadD1 complementation strain exhibited a phenotype similar to the wild-type strain. Note that quinolone resistance can result from changes in bacterial membrane permeation (Neu, 1992). The free acid of nalidixic acid, for instance, is easily dissolved in chloroform but only slightly soluble in water, indicating that both the polarity of quinolone in the medium and the composition of phospholipids (i.e., the proportions of different fatty acids) may influence the diffusion of quinolone through the membrane. To analyze changes in phospholipids, we assayed the fatty acid composition of the cells using GC-MS. The phospholipid fatty acids in $H$. parasuis are composed mainly of palmitic acid, stearic acid, palmitoleic acid, myristic acid and oleic acid (Figure 6). However, no significant differences were observed in fatty acid composition between the wild-type strain and the mutants (Table 7). Hence, we propose that deleting fadD1 might affect the amount of all lipids rather than the overall composition.

\section{DISCUSSION}

Increases in drug-resistant pathogenic bacteria have created an urgent need for new antibiotics. Type II fatty acid synthesis (FASII) is essential for the formation of cellular membranes in bacteria. There are significant differences between 


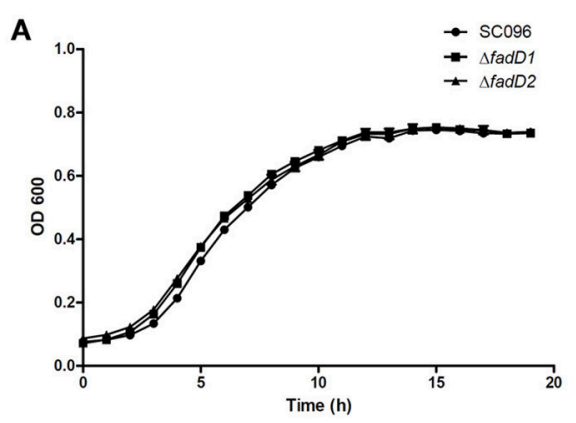

D

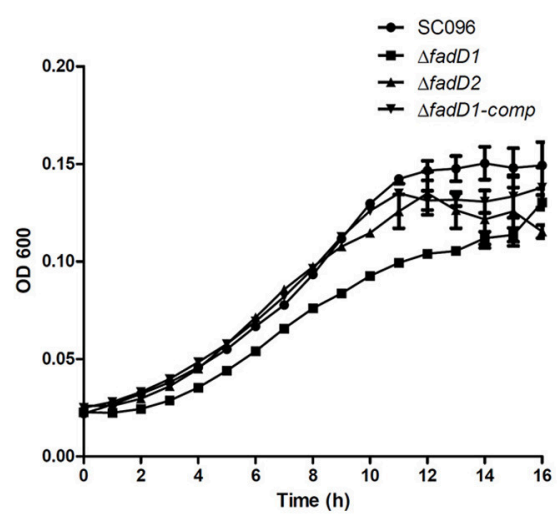

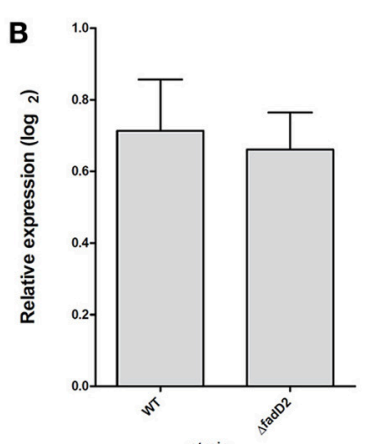

$\mathbf{E}$

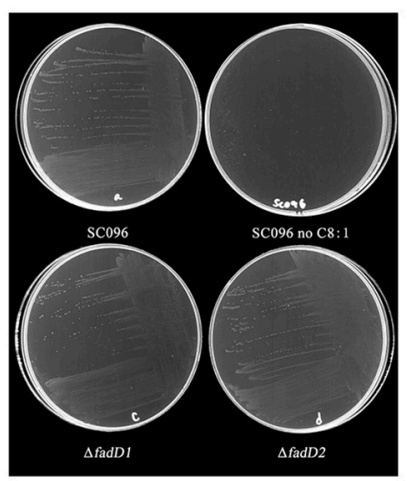

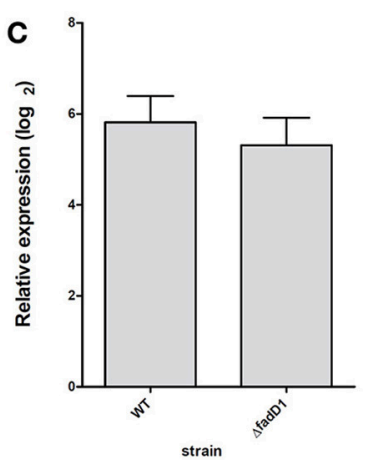

$\mathbf{F}$

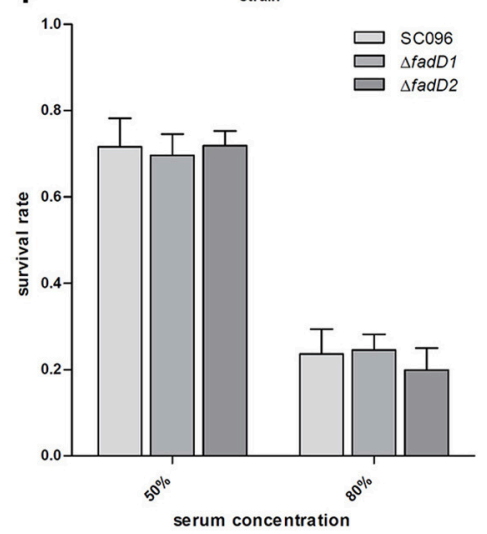

FIGURE 5 | Analysis of growth, the expression level of $\mathbf{f a d} \boldsymbol{D}$ genes and serum resistance of $\boldsymbol{H}$. parasuis fadD mutants. (A) The growth curve of wild type and mutants grown in TSA medium with 5\% newborn bovine serum and NAD. Only negligible changes in the growth rates were detected. (B) Real-time PCR analysis of fadD1 transcription in SC096 or $\triangle$ fadD2. (C) Real-time PCR analysis of fadD2 transcription in SC096 or $\triangle$ fadD1. Error bars represent the standard deviation from three independent experiments. No significant changes in transcription were observed in real-time PCR assays. (D) The growth curve of SC096, fadD mutants and fadD1 complementation strain grown in chemical defined medium with the addition of oleic acid. (E) The growth of SC096 and its fadD mutants on chemically defined medium plates in either the presence or the absence of oleic acid. (F) Survival of SC096 and fadD mutant strains in 50 or $80 \%$ porcine sera. The data represent means standard errors $(n=3)$ and no statistical difference was detected.

TABLE 6 | Susceptibilities of $\boldsymbol{H}$. parasuis strains to antimicrobials.

\begin{tabular}{|c|c|c|c|c|c|c|c|c|c|c|c|c|c|c|c|c|c|c|}
\hline \multirow{2}{*}{$\begin{array}{l}\text { Strain and relevant } \\
\text { phenotype(s) }\end{array}$} & \multicolumn{17}{|c|}{$\operatorname{MIC}(\mu \mathrm{g} / \mathrm{ml})^{a}$} & \multirow[b]{2}{*}{ PB } \\
\hline & LVX & ENR & NAL & CIP & ERY & AMP & EFT & GEN & AMK & CTX & KAN & STR & SMZ & TMP & DOX & TET & FF & \\
\hline SC096 & 1 & 1 & 64 & 1 & 1 & 0.125 & $\leq 0.016$ & 2 & 16 & $\leq 0.016$ & 8 & 16 & $\geq 512$ & 4 & 0.25 & 0.5 & 2 & 0.032 \\
\hline$\Delta f a d D 1$ & 0.25 & 0.25 & 16 & 0.25 & 0.25 & 0.125 & $\leq 0.016$ & 32 & 4 & $\leq 0.016$ & 2 & 8 & $\geq 512$ & 4 & 0.25 & 0.25 & 1 & 0.032 \\
\hline$\triangle f a d D 2$ & 1 & 1 & 64 & 1 & 1 & 0.125 & $\leq 0.016$ & 1 & 16 & $\leq 0.016$ & 32 & 8 & $\geq 512$ & 4 & 0.25 & 0.25 & 2 & 0.032 \\
\hline
\end{tabular}

a LVX, levofloxacin; ENR, enrofloxacin; NAL, nalidixic acid; CIP, ciprofloxacin; ERY, erythromycin; AMP, ampicillin; EFT, ceftiofur; GEN, gentamicin; AMK, amikacin; CTX, cefotaxime; KAN, kanamycin; STR, streptomycin; SMZ, sulfamethoxazole; TMP, trimethoprim; DOX, doxycycline; TET, tetracycline; FF, florfenicol; PB, polymyxin B.

mammalian and bacterial cells that make FASII enzymes efficient targets of antibacterial agents (Campbell and Cronan, 2001). There are currently many examples of natural or artificial products, including cerulenin, cephalochromin and triclosan, that have evolved to target FASII enzymes (Zhang et al., 2006). FASII inhibition cannot be bypassed in many bacteria because they cannot obtain essential fatty acids from the host (Yao and Rock, 2015). However, some pathogens can down-regulate FASII enzymes when exogenous fatty acids are present, allowing them to evade therapeutics. Previous studies have shown that exogenous fatty acids fully bypass inhibition by this pathway under both in vitro and in vivo conditions in gram-positive pathogens (Brinster et al., 2009). Nevertheless, few studies have focused on acyl-CoA synthetase (FACS) as a target for drug discovery because it may be not essential for the survival of many pathogens. Here, we present evidence showing that fadDs (long-chain FACS genes) are essential for $H$. parasuis SC096 survival 


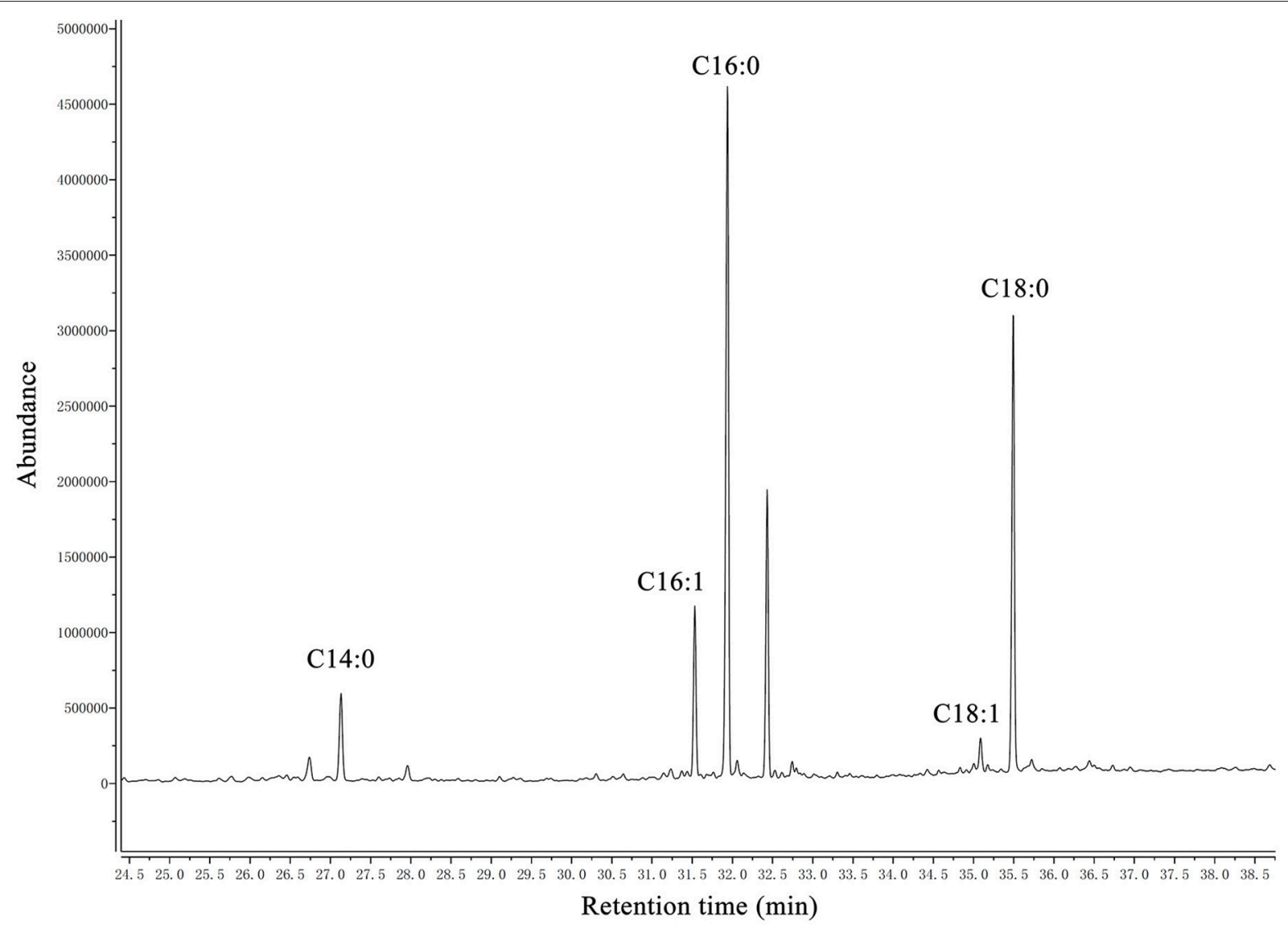

FIGURE 6 | GC-MS chromatogram of analysis of free fatty acids extracted from phospholipid of $\boldsymbol{H}$. parasuis Sc096. The fatty acids were derivatized to their methyl esters and then analyzed by gas chromatography-mass spectroscopy. C14:0, C16:0, C16:1, C18:1, and C18:0 are abbreviations of myristic acid, palmitic acid, palmitoleic acid, oleic acid and stearic acid, respectively.

TABLE 7 | Fatty acid composition of phospholipid extracts from H. parasuis fadD mutant strains grown in TSB medium.

\begin{tabular}{|c|c|c|c|c|c|}
\hline \multirow[t]{2}{*}{ Fatty acid ${ }^{a}$} & \multicolumn{5}{|c|}{ Strains } \\
\hline & SC096 & $\Delta f a d D 1$ & $\Delta$ fadD2 & $\Delta$ fadD1-comp & $\Delta$ fadD1-comp \\
\hline C14:0 & $6.9 \pm 0.5$ & $6.7 \pm 0.2$ & $5.3 \pm 0.1$ & $4.1 \pm 0.7$ & $4.8 \pm 0.4$ \\
\hline C16:0 & $48.4 \pm 3.2$ & $47.0 \pm 4.2$ & $48.8 \pm 3.9$ & $48.5 \pm 3.7$ & $48.1 \pm 2.9$ \\
\hline C16:1 & $11.9 \pm 1.8$ & $9.1 \pm 0.8$ & $9.3 \pm 0.9$ & $10.75 \pm 0.6$ & $11.0 \pm 1.0$ \\
\hline C18:0 & $29.5 \pm 4.2$ & $34.6 \pm 2.4$ & $34.7 \pm 3.3$ & $34.8 \pm 2.5$ & $34.2 \pm 2.8$ \\
\hline C18:1 & $3.3 \pm 0.3$ & $2.6 \pm 0.5$ & $2.0 \pm 0.8$ & $1.9 \pm 0.4$ & $1.9 \pm 0.6$ \\
\hline
\end{tabular}

a The fatty acids listed are those positively assigned by gas chromatographic data and with an abundance of greater than $0.1 \%$ in at least one sample. The data presented represent the mean $\pm S . D$. $(n=3)$ of results from three independent experiments.

(Figure 4 and Table 4), suggesting that these genes may be new targets for antibiotics and other drugs aimed at controlling Haemophilus bacterial infections. Notably, the human pathogen Haemophilus influenza also contains two predicted fadDs that share high homology with the fadDs in $H$. parasuis, although no studies have explored whether they are essential for $H$. influenza. In addition, the $H$. parasuis
fadD1 mutant showed increased sensitivity to quinolone antibiotics (Table 6), suggesting that if antibacterial agents could be developed against FadD, drug combinations including these agents may more effectively control these bacterial infections.

In this study, we also focused on characterizing $H$. parasuis FACSs. In E. coli, only two FACSs, FadD and FadK, have been identified (Black et al., 1992; Morgan-Kiss and Cronan, 2004), whereas at least six FadD homolog with a wide range of fatty acid activity have been described in Pseudomonas aeruginosa (Zarzycki-Siek et al., 2013). The diversity of substrates observed across different FACSs may be due to the evolution of bacteria in different ecological niches. To colonize such diverse environments, bacteria must rapidly adjust their metabolism and defense systems (DiRusso et al., 1999). Unlike many other bacteria, $H$. parasuis has only two FACSs for long-chain activation (Figure 3). The essential nature of $H$. parasuis fadDs may have resulted from adaptations to the host environment that occurred over a long period of evolutionary time. Our results demonstrate that host phospholipids may be vital nutrients to bacteria during $H$. parasuis infection. Additionally, FadD2 activity is sufficient to promote fadD1 mutant growth 


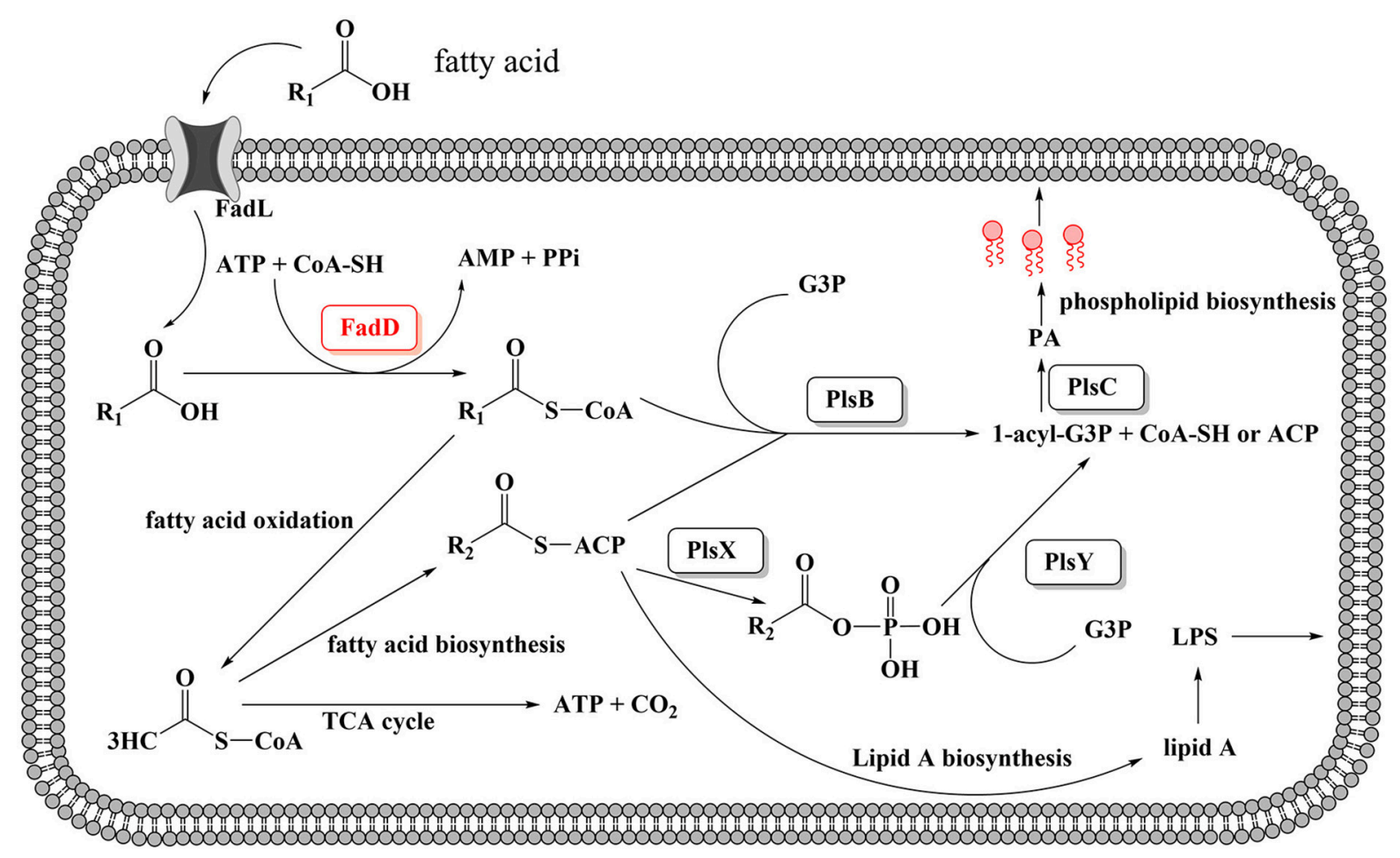

FIGURE 7 | Proposed model for exogenous fatty acid utilization by $\boldsymbol{H}$. parasuis FadDs. Exogenous fatty acids can be transported through the membrane by an outer membrane protein, for instance, FadL protein. Fatty acids then can be activated by acyl-CoA synthetase and converted to acyl-CoA. For $H$. parasuis, the enzymes may be FadD1 and FadD2. Subsequently, acyl-CoA can either be degraded to acetyl-CoA through $\beta$-oxidation or be utilized by PIsB and PlsC to synthesize the phospholipids then incorporate to the membrane. It should be noted that endogenous fatty acid is essential and can be synthesized from acetyl-CoA by FAS, then used for lipid A or phospholipids biosynthesis.

without phenotypic differences in rich medium (Figure 5A). The existence of fadD2 seems to be a supplementary mechanism rather than a strategy for wide-range fatty acid utilization, as the substrate specificity of these two FACSs is similar, although FadD2 exhibited lower activity (Figure 3). Palmitic acid may be a preferred fatty acid substrate according to our data, although a more extensive assessment of potential substrates of FadD2 is required to verify this. It is important to note that the activity of FACS in vivo may be determined not only by enzymatic properties but also by expression level. The result that FadD2 can support the growth of a fadD1 mutant without obvious differences may due to a higher expression level of FadD2 compared with FadD1.

A schematic diagram illustrates the exogenous fatty acid metabolism (Figure 7). It must be noted that while exogenous fatty acids can be incorporated into membrane phospholipids, they can also be converted to acetyl-CoA, which is a precursor for de novo fatty acid biosynthesis. There is no pathway through which exogenous fatty acids can be converted into $\beta$ hydroxyacyl-ACP, which is required for lipid A biosynthesis; lipid A is the core component of LPS, which is required for bacterial survival (Yao and Rock, 2015). Previous studies have shown that lipooligosaccharide (LOS) is an H. parasuis virulence factor. Mutants with truncated LOSs exhibit decreased resistance to complement-mediated killing in serum (Xu et al., 2013; Zhou et al., 2016). The results presented in this report demonstrate that the wild-type strain SC096 cannot grow on chemically defined medium without the addition of fatty acids (Figure 5E). Fatty acids and acyl-CoA are therefore indispensable, even though other carbon sources, such as amino acids and vitamins, are present in this medium. Exogenous fatty acids may be mainly converted to acetyl-CoA rather than directly transferred to the membrane. This process is followed by the rebuilding of phospholipids and lipid A via the FAS pathway. Hence, the fatty acid composition of phospholipids or lipid A may be determined by FAS but not the types or amount of exogenous fatty acids. This may explain why deleting fadD1 had no significant effect on fatty acid composition or serum resistance (Figure 5F and Table 7). Further studies are needed to confirm how disruptions in FadDs affect the $H$. parasuis membrane and LOS.

\section{CONCLUSIONS}

In summary, we identified two FACS homolog and determined the substrate specificities of each FadD in $H$. parasuis. Mutant and site-directed mutagenesis-generated strains were used to show that either $f a d D 1$ or $f a d D 2$ is required for survival in $H$. parasuis. Moreover, growth on chemical defined medium was 
affected in this bacterium by the deletion of fadD1. Finally, the fadD1 mutant showed increased sensitivity to quinolone antibiotics.

\section{AUTHOR CONTRIBUTIONS}

SF: Performed research, analyzed data, and wrote the paper. CX: Performed research. KY: Helped with experiment. HW: Analyzed data. HF: Funded research and analyzed data. ML: Funded research and analyzed data.

\section{REFERENCES}

Baba, T., Ara, T., Hasegawa, M., Takai, Y., Okumura, Y., Baba, M., et al. (2006). Construction of Escherichia coli K-12 in-frame, single-gene knockout mutants: the Keio collection. Mol. Syst. Biol. 2:2006.02008. doi: 10.1038/msb4100050

Baba, T., Huan, H. C., Datsenko, K., Wanner, B. L., and Mori, H. (2008). The applications of systematic in-frame, single-gene knockout mutant collection of Escherichia coli K-12. Methods Mol. Biol. 416, 183-194. doi: 10.1007/978-1-59745-321-9_12

Bi, H., Yu, Y., Dong, H., Wang, H., and Cronan, J. E. (2014). Xanthomonas campestris $\mathrm{RpfB}$ is a fatty Acyl-CoA ligase required to counteract the thioesterase activity of the RpfF diffusible signal factor (DSF) synthase. Mol. Microbiol. 93, 262-275. doi: 10.1111/mmi.12657

Black, P. N., and DiRusso, C. C. (2003). Transmembrane movement of exogenous long-chain fatty acids: proteins, enzymes, and vectorial esterification. Microbiol. Mol. Biol. Rev. 67, 454-472. doi: 10.1128/MMBR.67.3.454-472.2003

Black, P. N., DiRusso, C. C., Metzger, A. K., and Heimert, T. L. (1992). Cloning, sequencing, and expression of the $f a d D$ gene of Escherichia coli encoding acyl coenzyme A synthetase. J. Biol. Chem. 267, 25513-25520.

Brinster, S., Lamberet, G., Staels, B., Trieu-Cuot, P., Gruss, A., and Poyart, C. (2009). Type II fatty acid synthesis is not a suitable antibiotic target for Gram-positive pathogens. Nature 458, 83-86. doi: 10.1038/nature07772

Campbell, J. W., and Cronan, J. E. Jr. (2001). Bacterial fatty acid biosynthesis: targets for antibacterial drug discovery. Annu. Rev. Microbiol. 55, 305-332. doi: 10.1146/annurev.micro.55.1.305

Costa-Hurtado, M., and Aragon, V. (2013). Advances in the quest for virulence factors of Haemophilus parasuis. Vet. J. 198, 571-576. doi: 10.1016/j.tvjl.2013.08.027

Datsenko, K. A., and Wanner, B. L. (2000). One-step inactivation of chromosomal genes in Escherichia coli K-12 using PCR products. Proc. Natl. Acad. Sci. U.S.A. 97, 6640-6645. doi: 10.1073/pnas.120163297

Dennis, J. J., and Zylstra, G. J. (1998). Plasposons: modular self-cloning minitransposon derivatives for rapid genetic analysis of gram-negative bacterial genomes. Appl. Environ. Microbiol. 64, 2710-2715.

DiRusso, C. C., Black, P. N., and Weimar, J. D. (1999). Molecular inroads into the regulation and metabolism of fatty acids, lessons from bacteria. Prog. Lipid Res. $38,129-197$.

Falkow, S., Isberg, R. R., and Portnoy, D. A. (1992). The interaction of bacteria with mammalian cells. Annu. Rev. Cell Biol. 8, 333-363. doi: 10.1146/annurev.cb.08.110192.002001

Gulick, A. M. (2009). Conformational dynamics in the Acyl-CoA synthetases, adenylation domains of non-ribosomal peptide synthetases, and firefly luciferase. ACS Chem. Biol. 4, 811-827. doi: 10.1021/cb900156h

Guzman, L. M., Belin, D., Carson, M. J., and Beckwith, J. (1995). Tight regulation, modulation, and high-level expression by vectors containing the arabinose PBAD promoter. J. Bacteriol. 177, 4121-4130

Hill, C. E., Metcalf, D. S., and MacInnes, J. I. (2003). A search for virulence genes of Haemophilus parasuis using differential display RT-PCR. Vet. Microbiol. 96, 189-202. doi: 10.1016/S0378-1135(03)00212-8

Kameda, K., and Nunn, W. D. (1981). Purification and characterization of acyl coenzyme A synthetase from Escherichia coli. J. Biol. Chem. 256, 5702-5707.

Kang, Y., Zarzycki-Siek, J., Walton, C. B., Norris, M. H., and Hoang, T. T. (2010). Multiple FadD acyl-CoA synthetases contribute to differential fatty acid

\section{ACKNOWLEDGMENTS}

This work was supported by the Public Agriculture Specific Research Program (Grant No. 201303034).

\section{SUPPLEMENTARY MATERIAL}

The Supplementary Material for this article can be found online at: http://journal.frontiersin.org/article/10.3389/fcimb. 2017.00072/full\#supplementary-material

degradation and virulence in Pseudomonas aeruginosa. PLoS ONE 5:e13557. doi: 10.1371/journal.pone.0013557

Lucas, R. L., Lostroh, C. P., DiRusso, C. C., Spector, M. P., Wanner, B. L., and Lee, C. A. (2000). Multiple factors independently regulate hilA and invasion gene expression in Salmonella enterica serovar typhimurium. J. Bacteriol. 182, 1872-1882. doi: 10.1128/JB.182.7.1872-1882.2000

Metcalf, D. S., and MacInnes, J. I. (2007). Differential expression of Haemophilus parasuis genes in response to iron restriction and cerebrospinal fluid. Can. J. Vet. Res. 71, 181-188.

Morgan-Kiss, R. M., and Cronan, J. E. (2004). The Escherichia coli fadK (ydiD) gene encodes an anerobically regulated short chain acyl-CoA synthetase. J. Biol. Chem. 279, 37324-37333. doi: 10.1074/jbc.M405233200.

Murphy, T. F., and Brauer, A. L. (2011). Expression of urease by Haemophilus influenzae during human respiratory tract infection and role in survival in an acid environment. BMC Microbiol. 11:183. doi: 10.1186/1471-2180-11-183

Neu, H. C. (1992). Quinolone antimicrobial agents. Annu. Rev. Med. 43, 465-486. doi: 10.1146/annurev.me.43.020192.002341

Oliveira, S., and Pijoan, C. (2004). Haemophilus parasuis: new trends on diagnosis, epidemiology and control. Vet. Microbiol. 99, 1-12. doi: 10.1016/j.vetmic.2003.12.001

Pace, J., Hayman, M. J., and Galan, J. E. (1993). Signal transduction and invasion of epithelial cells by S. typhimurium. Cell 72, 505-514.

Ray, S., Chatterjee, E., Chatterjee, A., Paul, K., and Chowdhury, R. (2011). A fadD mutant of Vibrio cholerae is impaired in the production of virulence factors and membrane localization of the virulence regulatory protein TcpP. Infect. Immun. 79, 258-266. doi: 10.1128/IAI.00663-10

Rock, C. O., and Jackowski, S. (2002). Forty years of bacterial fatty acid synthesis. Biochem. Biophys. Res. Commun. 292, 1155-1166. doi: 10.1006/bbrc.2001.2022

Schafer, A., Tauch, A., Jager, W., Kalinowski, J., Thierbach, G., and Puhler, A. (1994). Small mobilizable multi-purpose cloning vectors derived from the Escherichia coli plasmids pK18 and pK19: selection of defined deletions in the chromosome of Corynebacterium glutamicum. Gene 145, 69-73.

Weeks, G., Shapiro, M., Burns, R. O., and Wakil, S. J. (1969). Control of fatty acid metabolism. I. Induction of the enzymes of fatty acid oxidation in Escherichia coli. J. Bacteriol. 97, 827-836.

Weimar, J. D., DiRusso, C. C., Delio, R., and Black, P. N. (2002). Functional role of fatty acyl-coenzyme A synthetase in the transmembrane movement and activation of exogenous long-chain fatty acids. Amino acid residues within the ATP/AMP signature motif of Escherichia coli FadD are required for enzyme activity and fatty acid transport. J. Biol. Chem. 277, 29369-29376. doi: 10.1074/jbc.M107022200

White, S. W., Zheng, J., Zhang, Y. M., and Rock (2005). The structural biology of type II fatty acid biosynthesis. Annu. Rev. Biochem. 74, 791-831. doi: 10.1146/annurev.biochem.74.082803.133524

Xu, C., Zhang, L., Zhang, B., Feng, S., Zhou, S., Li, J., et al. (2013). Involvement of lipooligosaccharide heptose residues of Haemophilus parasuis SC096 strain in serum resistance, adhesion and invasion. Vet. J. 195, 200-204. doi: 10.1016/j.tvjl.2012.06.017

Yao, J., and Rock, C. O. (2015). How bacterial pathogens eat host lipids: implications for the development of fatty acid synthesis therapeutics. J. Biol. Chem. 290, 5940-5946. doi: 10.1074/jbc.R114.636241

Zarzycki-Siek, J., Norris, M. H., Kang, Y., Sun, Z., Bluhm, A. P., McMillan, I. A., et al. (2013). Elucidating the Pseudomonas aeruginosa fatty acid degradation 
pathway: identification of additional fatty acyl-CoA synthetase homologues. PLoS ONE 8:e64554. doi: 10.1371/journal.pone.0064554

Zhang, B., Feng, S., Xu, C., Zhou, S., He, Y., Zhang, L., et al. (2012). Serum resistance in Haemophilus parasuis SC096 strain requires outer membrane protein P2 expression. FEMS Microbiol. Lett. 326, 109-115. doi: 10.1111/j.1574-6968.2011.02433.x

Zhang, Y. M., and Rock, C. O. (2008a). Membrane lipid homeostasis in bacteria. Nat. Rev. Microbiol. 6, 222-233. doi: 10.1038/nrmicro1839

Zhang, Y. M., and Rock, C. O. (2008b). Thematic review series: Glycerolipids. Acyltransferases in bacterial glycerophospholipid synthesis. J. Lipid Res. 49, 1867-1874. doi: 10.1194/jlr.R800005-JLR200

Zhang, Y. M., White, S. W., and Rock, C. O. (2006). Inhibiting bacterial fatty acid synthesis. J. Biol. Chem. 281, 17541-17544. doi: 10.1074/jbc.R600004200

Zhou, Q., Feng, S., Zhang, J., Jia, A., Yang, K., Xing, K., et al. (2016). Two glycosyltransferase genes of Haemophilus parasuis SC096 implicated in lipooligosaccharide biosynthesis, serum resistance, adherence, and invasion. Front. Cell. Infect. Microbiol. 6:100. doi: 10.3389/fcimb.2016.00100
Zhu, L., Bi, H., Ma, J., Hu, Z., Zhang, W., Cronan, J. E., et al. (2013). The two functional enoyl-acyl carrier protein reductases of Enterococcus faecalis do not mediate triclosan resistance. MBio 4:e00613. doi: 10.1128/mBio.00613-13

Zou, Y., Feng, S., Xu, C., Zhang, B., Zhou, S., Zhang, L., et al. (2013). The role of galU and galE of Haemophilus parasuis SC096 in serum resistance and biofilm formation. Vet. Microbiol. 162, 278-284. doi: 10.1016/j.vetmic.2012.08.006

Conflict of Interest Statement: The authors declare that the research was conducted in the absence of any commercial or financial relationships that could be construed as a potential conflict of interest.

Copyright $\odot 2017$ Feng, Xu, Yang, Wang, Fan and Liao. This is an open-access article distributed under the terms of the Creative Commons Attribution License (CC BY). The use, distribution or reproduction in other forums is permitted, provided the original author(s) or licensor are credited and that the original publication in this journal is cited, in accordance with accepted academic practice. No use, distribution or reproduction is permitted which does not comply with these terms. 